\title{
Water Resources Management \\ Dealing with uncertainty in decision-making for drinking water supply systems exposed to extreme events \\ --Manuscript Draft--
}

\begin{tabular}{|c|c|}
\hline Manuscript Number: & WARM-D-17-00705R1 \\
\hline Keywords: & $\begin{array}{l}\text { Emergency management; Drinking water supply systems; Bayesian Belief Networks; } \\
\text { uncertainty analysis; Decision support system }\end{array}$ \\
\hline \multicolumn{2}{|l|}{$\begin{array}{l}\text { Corresponding Author Secondary } \\
\text { Information: }\end{array}$} \\
\hline First Author: & Alessandro Pagano, Ph.D. \\
\hline \multicolumn{2}{|l|}{ First Author Secondary Information: } \\
\hline \multirow[t]{5}{*}{ Order of Authors: } & Alessandro Pagano, Ph.D. \\
\hline & Irene Pluchinotta \\
\hline & Raffaele Giordano \\
\hline & Anna Bruna Petrangeli \\
\hline & Umberto Fratino \\
\hline
\end{tabular}


1 Dealing with uncertainty in decision-making for drinking water supply systems exposed to 1

5 Alessandro Pagano ${ }^{1, *}$, Irene Pluchinotta ${ }^{2}$, Raffaele Giordano ${ }^{1}$, Anna Bruna Petrangeli ${ }^{1}$, Umberto alessandro.pagano@ba.irsa.cnr.it; petrangeli@irsa.cnr.it; raffaele.giordano@cnr.it; michele.vurro@ba.irsa.cnr.it

2 LAMSADE - CNRS, Univ. Paris-Dauphine, PSL Research Univ. 


\section{Abstract

The availability and the quality of drinking water are key requirements for the well-being and the safety of a community, both in ordinary conditions and in case of disasters. Providing safe drinking water in emergency contributes to limit the intensity and the duration of crises, and is thus one of the main concerns for decision-makers, who operate under significant uncertainty. The present work proposes a Decision Support System for the emergency management of drinking water supply systems, integrating: i) a vulnerability assessment model based on Bayesian Belief Networks with the related uncertainty assessment model; ii) a model for impact, and related uncertainty assessment, based on Bayesian Belief Networks. The results of these models are jointly analyzed, providing decision-makers with a ranking of the priority of intervention. A GIS interface $(G-N e t)$ is developed to manage both input spatial information and results. The methodology is implemented in L'Aquila case study, discussing the potentialities associated to the use of the tool dealing with information and data uncertainty.

Keywords: Emergency management; Drinking water supply systems; Bayesian Belief Networks; Uncertainty Analysis; Decision Support System 
Modern societies highly rely on infrastructures, which provide critical services and guarantee the quality of life for citizens (Zhao et al. 2016). The increase in both frequency and intensity of extreme events contributes to create additional challenges to the infrastructure providers (Eidsvig et al. 2017). Particularly, water supply infrastructures are essential for health, sanitary and economic reasons and, consequently, there is high pressure on water organizations to provide customers with a continual and efficient water supply (Mala-Jetmarova et al. 2017).

Several approaches are available for protecting water supply infrastructures from a wide variety of stresses, either supporting system performances assessment in case of extreme events (EPA 2015) or driving the selection of suitable actions for vulnerabilities mitigation (Fragiadakis et al. 2013). Methods typically vary with the type of system, the aim of the analysis, and the available information. A broad classification is into qualitative, semi-quantitative and quantitative approaches (Pagano et al. 2014a; Eidsvig et al. 2017). Quantitative tools require detailed data and a high computational burden, but provide reliable numerical outcomes for decision-makers (Fragiadakis et al 2013, Diao et al. 2016). Qualitative approaches support ranking risk levels, screening and identifying critical scenarios (Eidsvig et al. 2017), based on the use of classes (e.g. 'high', 'medium', 'low'). Semi-quantitative techniques (e.g. probabilistic methods such as Bayesian Belief Networks) guarantee a compromise between such classes.

One of the most challenging tasks in these methods is uncertainty management. Uncertainty represents the lack of exact knowledge, which is inherently associated to water supply systems planning, design and operation (Tanyimboh 2017). Specifically, the uncertainties related to emergency onset and evolution (Perng and Buscher 2015) as well as the difficulty in collecting reliable data and the ambiguity in the understanding of specific phenomena should be properly considered. These issues deeply affect the capability to identify optimal decisions for emergency management (Pagano et al. 2014b, Gaudard and Romerio 2015). Enhancing the understanding of 
uncertainties could support developing a representative picture of the current knowledge and its potential deficiencies (Uusitalo et al. 2015, van der Keur et al. 2016).

Bayesian Belief Networks (BBNs) have shown several useful features to support decision-making under uncertainty for water supply systems (Molina et al. 2011). BBNs allow the integration of various types of information combining qualitative and quantitative aspects (Gonzalez-Redin et al. 2016, Phan et al. 2016). They support reasoning from uncertain evidence to uncertain conclusion (John et al. 2016), treating both data and model uncertainty (Marcot 2012, Uusitalo et al. 2015, Gonzalez-Redin et al. 2016).

Within this framework, the present work describes a Decision Support System (DSS) for the emergency management of drinking water supply infrastructures. The DSS is based on the integration of: i) a probabilistic vulnerability assessment model, based on BBNs, to identify the most critical elements of the infrastructural system; ii) the associated uncertainty estimate; iii) a BBN-based model for impact assessment; iv) the associated uncertainty estimate. The most relevant innovation of the present work is twofold. Firstly, the definition of a methodology to perform a joint vulnerability and impact assessment of infrastructural failure, with an explicit uncertainty analysis. This is a crucial requisite in the definition of a set of decision-makers' preferences to support defining a priority of actions in emergency. Secondly, overcoming one of the main limits of BBNs, which are not inherently characterized by a spatial nature, a GIS interface $(G-N e t)$ was built to support the management of input spatial information and results visualization. The DSS was developed with the cooperation of the Italian Department of Civil Protection (DPC), tested with several Italian water utilities (Acquedotto Pugliese S.p.A., Gran Sasso Acqua S.p.A. and AIMAG S.p.A.), and implemented in a relevant case study: L'Aquila (Italy) earthquake in 2009.

The paper is structured as follows. After the present introduction, Section 2 provides an overview of BBNs features and applications. Section 3 describes the architecture of the developed tool. Section 4 
1 discusses the relevance of L'Aquila case study, while section 5 includes a discussion on the main

1

2 3

4

53

7

8 10

\section{Methodological background: Bayesian Belief Networks}

BBNs combine graph theory and probability theory, consisting of directed acyclic graphs and associated joint probability distribution (Pearl 1988). The graph nodes represent variables, whereas the edges represent conditional dependencies. The strength of the dependency is represented by conditional probabilities: each variable $X_{i}$ is associated to a probability function $P\left(X_{i} \mid p_{a i}\right)$ that takes as input $p_{a i}$, i.e. a set of predecessors of $X_{i}$ which make $X_{i}$ independent on all other predecessors. Variables that are judged as direct causes of $X_{i}$ satisfy this property, and are the parent variables of the node. BBNs thus allow the probabilistic representation of interactions between variables (Pearl 1988, Phan et al. 2016). The importance of BBNs is mainly related to the ability to coordinate bidirectional inferences, supporting the representation and analysis of uncertain knowledge as well as different modes of reasoning (Pearl 1988).

BBNs have become an increasingly popular modelling technique to deal with complexity and uncertainty and several studies focused on the potentialities of BBNs to support decision-making in several emergency conditions (e.g. Sobradelo et al. 2015, Wu et al. 2017). Referring specifically to water supply infrastructures exposed to external stresses, BBNs were mainly used to build models for pipe breaks using learning from past breaks, integrating multiple kinds of data and modeling explicitly the dependencies, using probabilities updates and a representation of uncertainty (Francis et al. 2014, Kabir et al. 2015, Kabir et al. 2016).

A wide scientific literature underlined that BBNs are able to support: the integration of various types of information (e.g. analytical models, expert knowledge, literature and historical data) (GonzalezRedin et al. 2016, Phan et al. 2016), the possibility of reasoning from uncertain evidence to uncertain conclusions (John et al. 2016), the explicit treatment of uncertainties (Uusitalo 2007, Uusitalo et al. 
2015, Gonzalez-Redin et al. 2016). Furthermore, BBNs are also flexible enough to support a revision of probabilities in the light of additional information or observations availability.

BBNs have also some limitations. Firstly, nodes are often discretized with only a few states and in qualitative terms (e.g. 'high' or 'low'), providing a coarse representation (Uusitalo, 2007). Secondly, the BBNs structure is linear and static, and does not directly account for the analysis of feedback loops and dynamic issues (Uusitalo, 2007). Furthermore, BBNs do not natively provide a spatial representation of variables.

Specifically referring to the last issue, Johnson et al. (2011) identified four ways to integrate GIS and BBNs: i) GIS input to BBN, when GIS layers are used as input nodes; ii) GIS input to, and output from $\mathrm{BBN}$, in case GIS is also used to visualize the output of a BBN; iii) BBN and GIS complex interactions; iv) BBN and GIS within a larger framework, where BBNs model one factor and GIS models other factors. Integrated methodologies based on BBNs and GIS were recently proposed (e.g. Landuyt et al. 2015, Gonzalez-Redin et al. 2016, Molina et al. 2016, Liu et al. 2016), showing remarkable potentialities. Uncertainty maps can be developed as well, as discussed by Landuyt et al. (2015).

\section{Model description}

The present work describes a DDS developed for decision-makers involved in the management of drinking water supply infrastructures under emergency conditions.

The DSS is based on the integration of:

- A probabilistic vulnerability assessment model, based on BBN, for the infrastructural system. The model is integrated in a GIS tool $(G-N e t)$ in order to facilitate data input and to provide a geographical visualization of results (Section 3.1). 
- An uncertainty analysis related to the results of the vulnerability assessment model, used to analyze the impacts of the available knowledge (and existing gaps) on the results (Section 3.2).

- A BBN-based probabilistic model for impact assessment, useful to quantify the magnitude of the impacts of an event (Section 3.3).

- An uncertainty analysis related to the results of the impacts assessment model (Section 3.3).

In the end, decision-making is supported through the definition of a ranking order among the elements of the network, based on the integration of information on infrastructural vulnerability, impacts and related uncertainties.

\subsection{G-Net tool for the spatial vulnerability assessment}

The first element of the DSS is a vulnerability assessment tool for drinking water supply infrastructures based on BBNs, whose conceptual structure is described in Pagano et al. (2014a). The tool is composed of a set of BBNs quantifying the vulnerability levels of drinking water supply systems from source to tap, with respect to physical (earthquakes, landslides) or CBR hazards (water contamination).

The following Fig. 1 shows the BBN used to analyze the physical vulnerability of water mains. It may be used either to assess the global vulnerability level, or the vulnerability associated to specific mechanisms (i.e. breaking, corrosion, joint extraction and security level). The variables in grey represent the 'parent' variables (input), whereas those in yellow are the 'child' variables (output).

Three main classes of data are included in the model: infrastructural data (e.g. diameter, material, thickness, etc.); environmental data (e.g. seismicity, soil mechanical characteristics, etc.); operative data (e.g. hydraulic variability, maintenance performed/scheduled, etc.). The outcome is, for each element of the network under investigation, a set of probability values associated to the states of specific output variables. Further details on model building are included in the Supplementary Material. 
Fig. $1 \mathrm{BBN}$ for the physical vulnerability assessment of water mains

It is worth mentioning that each pipe is analyzed independently, thus neglecting the role of structural or functional interconnections, dependencies and cascading effects (e.g. a vulnerable element might have impacts on the whole infrastructure downstream). This allows easily identifying the most vulnerable elements of the whole network (further details in Pagano et al. 2014a).

Based on the feedbacks obtained by the potential end-users, i.e. DPC and water utilities, a GIS interface was built, in order to facilitate spatial data processing and results representation. The toolbox G-Net consists of an expanded development of a GIS application supporting the vulnerability assessment tool. It is specifically designed to support the integration with $\mathrm{Netica}^{\mathrm{TM}}$ software by means of an automated procedure. The tool is composed of customized interfaces working in ArcGIS® software (by Esri) environment with wizards configured as interface between Netica ${ }^{\mathrm{TM}}$ and ArcGIS® .

The tool has been designed using open-source Python scripting language, fully supported by ArcGIS $®$ and able to extend the basic functionality of GIS and to automate the workflow (Tateosian 2015). A loosely-coupled integration strategy between ArcGIS $®$ and Netica ${ }^{\mathrm{TM}}$ was used. This means that the latter is not completely encapsulated within a GIS environment, but takes advantage of the database, the visualization and the analysis capabilities of a GIS (Karimi and Houston 1996, Johnson et al. 2011)

$G$-Net was developed both for the collection, analysis and attribution of spatial input data and for the visualization and mapping of the outcomes of the vulnerability assessment. Referring to the different classes of BBN-GIS interactions introduced above (Johnson et al. 2011), G-Net refers to the second category, which is 'GIS input to, and output from BBN'.

A schematic overview of the procedure carried out by the tool is shown in the Fig. 2. 
Figure 2. G-Net procedure for vulnerability assessment and mapping: (a) selection of the analysis to perform; (b) data association to the input variables; (c) input variables export procedure; (d) output vulnerability map.

$G$-Net firstly requires the selection of the subsystem to analyze, among all the elements of a drinking water infrastructure, both linear (e.g. water mains) and punctual (e.g. tanks, pumping systems, etc.). Secondly, the user should select the kind of analysis to carry out (Figure 2a), i.e. physical or CBR vulnerability assessment. Additional data related to the input variables in the BBN can be manually or automatically associated to the file (Figure $2 b$ ). If some data concerning a certain variable are not available, a uniform probability distribution is considered and the BBN propagates the related uncertainty up to the output variables.

Once the GIS pre-processing is complete, G-Net exports a table for the input variables in a format easily manageable by Netica ${ }^{\mathrm{TM}}$ (Figure 2c). Following the vulnerability assessment procedure in Netica $^{\mathrm{TM}}$, a table with modeling results can be imported again in GIS, and joined to the available file, through the same toolbox. Afterwards, the resulting BBN is shown in the vulnerability map (Figure $2 d)$.

\subsection{Uncertainty analysis}

The present section aims at defining a method to analyze and map the uncertainty associated to BBNs, supporting the identification of its root causes. Reference is made to the work by Marcot (2012), who suggested metrics for estimating model performances and uncertainty. Referring to BBNs, uncertainty pertains to the dispersion of Posterior Probability Distribution (PPD), i.e. the spread of alternative predictions.

Firstly, the sensitivity analysis (SA) supports determining the degree to which a variation in PPD is explained by other variables, and depicts the underlying probability structure of a model (Marcot 2012). It was performed with respect to the variable 'breaking vulnerability', and the results are proposed in the Table 1. The results of SA are also used for scenario analysis (see section 5). 
Table 1. Results of the sensitivity analysis performed with respect to the variable 'breaking

vulnerability'

\section{TABLE 1}

The more sensitive to a variable the model is, the more important is to collect related information. Having reliable data on key variables is a crucial requisite to reduce uncertainty.

Secondly, the uncertainty associated to BBNs is estimated using the Shannon entropy $H(X)$ referring to the output variable ('breaking vulnerability' for the vulnerability assessment model). It is defined as the average amount of information conveyed by a stochastic source of data. The concept of Shannon Entropy is fundamental in information theory and, besides sharing some intuition with Boltzmann's theory, some aspects are analogous to those used in statistical thermodynamics. The Shannon entropy can be used as a synthetic measure of uncertainty, related to the number of alternatives and characteristics of the probability distribution over the states of a random variable (Das 1999). It is expressed as follows, using a logarithmic form:

$$
H(X)=-\sum_{i=1}^{n} P\left(x_{i}\right) \log P\left(x_{i}\right)
$$

$H(X)$ measures the average information required in addition to the current knowledge to remove the ignorance associated to the probability distribution of $X$. If the current state of knowledge is complete, then $H(X)=0$. If it is total ignorance (uniform probability distribution), the additional information required to pin down an alternative is maximum. A normalized value of entropy can be calculated as $\bar{H}(X)=H(X) / H(X)_{\max }$. For the purposes of the present work, the Shannon entropy is used to estimate the uncertainty related to the main output variables (i.e. 'breaking vulnerability' and 'impacts').

\subsection{Impact assessment}

The levels and types of adverse impacts are the result of a physical event interacting with vulnerable elements. The aim of emergency managers is directly related to the reduction of impacts, both before 
and after a disaster occurs (McCormick 2016). Correctly assessing the impacts of an emergency is not a straightforward task, due to the complexity associated to a comprehensive analysis of costs and consequences (Sobradelo et al. 2015).

For the purpose of the present work, the impact assessment is performed through another BBN (Figure 3), based on the following key variables:

- 'Flow rate': measure of the service loss, depending on the number of users potentially affected. The values 'high', 'medium' and 'low' are defined considering whether the ratio between the local flow rate and the maximum upstream value is higher than 0.7 , between 0.3 and 0.7 or lower than 0.3 .

- 'Diameter': measure of the cost for repair, proportional to pipe diameter. The values 'high', 'medium' and 'low' are defined for each element considering whether the ratio between the local diameter and the maximum value is higher than 0.7 , between 0.3 and 0.7 or lower than 0.3 .

- 'Relevance': defines the presence of critical users and services (e.g. hospitals). The values 'high', 'medium' and 'low' are defined considering the importance of the services depending on the infrastructure.

- 'Redundancy': defines the presence of additional paths for water supply. The values 'Yes' and 'No' are defined considering the presence of other paths that can be activated.

\section{FIG 3}

Figure 3. BBN for impact assessment

\section{L'Aquila case study}

L'Aquila province (central Italy) was struck by a severe earthquake on 6 April 2009. Several damages to structures and infrastructures were detected over a broad area (Kongar et al. 2017). Referring to the water supply system, the major damage occurred on an important steel pipe (diameter $600 \mathrm{~mm}$; 
pressure 25-30 atm), which failed because crossing the surface trace of a fault activated during the earthquake (Pagano et al. 2017). The operation of the whole system was stopped in order to allow the restoration of infrastructural functionality and to limit the impacts of the multiple damages occurred in the urban distribution system. According to the interviews held with technicians involved in emergency operations, the fragmented and uncertain knowledge related to infrastructural conditions, particularly in the urban area, was a key limit during emergency operations. The available data were often not reliable and directly usable, since mainly deriving from personal experience, and thus difficult to share, visualize and integrate. Most of emergency operators acknowledged the lack of reliable infrastructural information as a main issue hampering the effectiveness of emergency management strategies.

\section{Results and discussion}

\subsection{Vulnerability assessment}

The main results of the vulnerability assessment procedure, performed through $G$-Net in L'Aquila case study, are represented in Figure 5(a) along with the results of the uncertainty assessment. These results are identified in the following as the 'BASE' scenario. The map plots the probability values associated to the state 'high' of the variable 'breaking vulnerability'.

The Figure 5(a) shows the presence of several elements having values of 'breaking vulnerability' from 'medium' to 'high'. Model predictions were tested comparing the results with the position of the main pipe breaks occurred during the earthquake. Particularly, the highest values of 'breaking vulnerability' were found for the pipe damaged in 2009 . Then, other elements characterized by a significantly high 'breaking vulnerability' were identified as well, and the result discussed with GSA S.p.A., resulting in a correspondence with some well-known vulnerabilities of the infrastructure.

\subsection{Uncertainty analysis and mapping}


1 Starting from the results of the SA (Section 3.2), an influence analysis was performed. It allows

evaluating (and comparing) the effects on PPD from selected input variables set to specific scenario values. Conducting influence runs can help reveal the degree to which individual or sets of input variables could affect output probabilities. This is helpful in a decision-setting, where management might prioritize activities to best effect desirable, or to avoid undesirable outcomes (Marcot 2012).

The following scenarios were analyzed and discussed:

- BEST Scenario: all the variables to their optimal state - i.e. minimizing the vulnerability of the system.

- WORST Scenario: all the variables to their worst state - i.e. maximizing the vulnerability of the system.

- UNCERTAIN Scenario: all the variables to an 'unknown' state - i.e. the input variables have uniform probability distribution, in case no information is available.

Three additional scenarios were built as well, changing the state of some variables according to the results of the SA. The variables modified in each scenario are identified in the Table 1.

- SENSIT (1). The scenario is built setting three key environmental variables to the worst state: 'seismicity', 'existing instabilities' and 'dynamic loads'. All the variables considered in this scenario represent external conditions, and thus their state cannot be improved.

- SENSIT (2). The scenario is built considering the positive impact of actions performed on variables that can be modified through specific strategies. These variables may be representative of both structural and operational aspects. In this scenario, a subset of variables is set to the best state.

- SENSIT (3). The scenario is built considering the four most influential variables, according to the sensitivity analysis, all set to the worst state. 
1 The results are summarized (according to Marcot 2012) in terms of PPD of the output variable 1

'breaking vulnerability' (Figure 4). The 'BEST', 'WORST' and 'UNCERTAIN' scenarios show an intuitive PPD for the output variable. The comparison between the scenarios 'SENSIT (3)' and 'SENSIT (1)' suggest that few variables, mainly related to environmental conditions, are highly influential on the result. From a practical point of view, this means that a deep knowledge of the environment in which a system is located (e.g. seismicity of the area, existing instabilities) is crucial for the reliable estimate of 'breaking vulnerability'. The Scenario 'SENSIT (2)' is indeed relevant in order to assess the impact of potential improvements on infrastructural and operational features. Although the effect on the output PPD is lower, acting on the infrastructure and changing operative conditions may contribute to reduce significantly the vulnerability level of the system.

\section{FIG 4}

Figure 4. Results of the influence analysis in the scenarios

The Shannon entropy was then used to produce uncertainty maps, as shown in Fig. 5. Referring to the 'BASE' scenario, the values of $H(X)$ were computed for the whole network and spatially plotted along with the results of the vulnerability assessment (Fig. 5a). The same procedure was used to map the impacts magnitude and the related uncertainty (Fig. 5b).

The relevance of $H(X)$ for uncertainty assessment was further tested through specific simulations, analyzing the impacts of the lack of important input information on the reliability of model results. The 'BASE' Scenario was built considering a full knowledge of the input variables required by the model. Referring also to Table 1, the following scenarios were created:

- $U(1)$ Scenario considers complete uncertainty for the input variables identified with (1) in Table 1. Three highly influential environmental variables (according to the SA): 'seismicity', 'existing instabilities' and 'dynamic loads', are treated as unknown. 
- $\mathrm{U}(2)$ Scenario considers complete uncertainty for the input variables identified with (2) in Table 1. Both structural and operative features are set to a uniform probability distribution.

- $U(3)$ Scenario considers uncertainty for the input variables identified with (3) in Table 1 and the four most relevant variables according to the SA are set as unknown.

The $H(X)$ was used in the cited scenarios, to quantify the cumulative uncertainty related to unknown inputs. Following the 'chain rule' for entropy, the global entropy of a group of random variables was computed as the sum of conditional entropies. The values of $H(X)$ are $0,0.067,0.012$ and 0.083 respectively for BASE, $U(1), U(2)$ and $U(3)$ scenarios. This suggests that although the scenario $U(2)$ is characterized by a higher number of unknown variables, their impact on modeling results is lower if compared to the key variables neglected in both $U(1)$ and $U(3)$ scenarios. Both $U(1)$ and $U(3)$ scenarios suggest that the knowledge related to environmental conditions is a key requirement to perform a reliable vulnerability assessment. Furthermore, referring particularly to the scenario U(3), the highest value of $H(X)$ is representative of a more critical condition, due to the highly uncertain set of available input data.

\subsection{Impact assessment}

The results of the impact assessment can be represented, as in the Figure 5b, based on the probability associated to the state 'high' of the variable 'impacts'. Both a numerical and a chromatic scale are used. As already discussed, the map represents also the associated uncertainty.

\section{FIG 5}

Figure 5. a) Results of vulnerability assessment and related uncertainty; b) Results of impacts assessment and related uncertainty.

\subsection{Recommendations for decision-makers}

The present section aims at supporting decision-makers in prioritizing the interventions on a drinking water supply infrastructure. The values of infrastructural vulnerability, the magnitude of the expected 
1 impacts, and the role of uncertainty are jointly taken into account. The network elements are

compared considering different combinations of 'vulnerability under uncertainty' and 'impacts under uncertainty'. Considering the drinking water supply infrastructure under analysis, each network element is characterized by the set of attributes $\mathcal{A}=\left\{\alpha_{1}, \alpha_{2}, \alpha_{1 u}, \alpha_{2 u}\right\}$, such that $\mathcal{A}_{\mathcal{L}}=$ $\left\{v_{h}, v_{m}, v_{l}, e_{h}, e_{m}, e_{l}, u_{1 h}, u_{1 m}, u_{1 l}, u_{2 h}, u_{2 m}, u_{2 l},\right\}$ represents the set of all possible values that the elements of $\mathcal{A}$ can take, over which a decision-maker has preferences. The attributes are:

- $\alpha_{1}$, vulnerability based on the state 'high' of the variable 'breaking vulnerability'. The possible values of the attribute are $\alpha_{1}=\left\{\operatorname{high}\left(v_{h}\right)\right.$, medium $\left(v_{m}\right)$, low $\left.\left(v_{l}\right)\right\}$;

$-\alpha_{2}$, impact assessment through the analysis of the exposure to the potential effects of failures represented by the values $\alpha_{2}=\left\{\operatorname{high}\left(e_{h}\right)\right.$, medium $\left(e_{m}\right)$, low $\left.\left(e_{l}\right)\right\}$;

- $\alpha_{1 u}$ and $\alpha_{2 u}$ uncertainty associated respectively to vulnerability and impact assessment, according to $\bar{H}(X), \alpha_{1 u}=\left\{\operatorname{high}\left(u_{1 h}\right)\right.$, medium $\left(u_{1 m}\right)$, low $\left.\left(u_{1 l}\right)\right\}$

and $\alpha_{2 u}=\left\{\right.$ high $\left(u_{2 h}\right)$, medium $\left(u_{2 m}\right)$, low $\left.\left(u_{2 l}\right)\right\}$.

Throughout this section, the symbol $>$ denotes a decision maker's preference relation, $x>y$ means that $x$ is preferred to $y$. The decision-makers have the following order of preferences: a higher value of vulnerability/exposure has priority compared to a lower one: $v_{h}>v_{m}>v_{l}$ and $e_{h}>e_{m}>e_{l}$. The preferences elicitation was performed through semi-structured interviews held with Civil Protection operators and engineers working for the local water utility. Considering the combination between the two attributes, the decision-makers should prioritize the highest possible value of $\alpha_{1}$ combined with the highest possible value of $\alpha_{2}: v_{h} e_{h}>v_{h} e_{m}>v_{m} e_{h}>v_{h} e_{l}>v_{m} e_{m}>v_{l} e_{h}>v_{m} e_{l}>v_{l} e_{m}>$ $v_{l} e_{l}$. However, as discussed in section 5.2, the 'uncertainty' is a key attribute that decision-makers take into account. Considering the preferences on the other attributes, a lower value of uncertainty associated respectively to vulnerability and impact assessment is preferred to a higher value: $u_{1 l} u_{2 l}>$ $u_{1 l} u_{2 m}>u_{1 m} u_{2 l}>u_{1 l} u_{2 h}>u_{1 m} u_{2 m}>u_{1 h} u_{2 l}>u_{1 m} u_{2 h}>u_{1 h} u_{2 m}>u_{1 h} u_{2 h}$. 
Accordingly to the preference statements, we obtain the following compact representation supporting the definition of a ranking order among the different potential 81 conditions:

$$
\begin{gathered}
v_{h} e_{h} u_{1 l} u_{2 l}>v_{h} e_{h} u_{1 l} u_{2 m}>v_{h} e_{h} u_{1 m} u_{2 l}>v_{h} e_{h} u_{1 l} u_{2 h}>v_{h} e_{h} u_{1 m} u_{2 m}>v_{h} e_{h} u_{1 h} u_{2 l}> \\
>v_{h} e_{h} u_{1 m} u_{2 h}>v_{h} e_{h} u_{1 h} u_{2 m}>v_{h} e_{h} u_{1 h} u_{2 h}>v_{h} e_{m} u_{1 l} u_{2 l}>v_{h} e_{m} u_{1 l} u_{2 m}>\cdots> \\
>\cdots>v_{l} e_{l} u_{1 h} u_{2 h}=r_{1}>r_{2}>r_{3}>\cdots>r_{81}
\end{gathered}
$$

Consequentially, in relation to the water supply network under analysis, we obtain the spatial representation of ranking as in the Fig. 6. The mapping of results allows decision-makers to identify the elements of the network where interventions should be primarily oriented either in emergency conditions or in ordinary management, to reduce the risk levels for the whole system.

\section{FIG 6}

Figure 6. Ranking of the network elements

\section{Conclusions}

This work describes a DSS for decision-making in the emergency management of drinking water supply systems. The methodology was implemented in L'Aquila case study. The model is composed of a BBN-based vulnerability assessment tool for drinking water supply infrastructures, with the related uncertainty analysis and a BBN-based model to estimate impacts magnitude, with the related uncertainty analysis. The tools are integrated in a comprehensive methodology, based on preferences orders, capable to jointly take into account all the previous information, and to define a ranking order among the elements of the infrastructural system. This ranking simply suggests a priority of action for decision-makers. Overcoming one of the main limitations of BBNs -i.e. the difficulties in performing spatial analyses- the development of a GIS interface $(G-N e t)$, for data structuring and results analysis, revealed highly useful to improve the effectiveness of the tool, helping in visualizing the outcomes, quantifying uncertainty, and identifying the final ranking. Future activities will be oriented mainly to the analysis of temporal aspects related to the dynamic evolution of system 
behavior (see e.g. Pagano et al. 2017) and to the implementation of models based on complexity

1

2
3 theory to support the analysis of interconnected systems.

\section{Acknowledgments}

The present research activity was developed within a research project funded by the Italian Department of Civil Protection ('Intesa Operativa del 19.12.2006 tra DPC e IRSA—Rep. 618).

\section{References}

Das B (1999) Representing Uncertainties Using Bayesian Networks. DSTO-TR-0918, DSTO Electronics and Surveillance Research Laboratory, Australia

Diao K, Sweetapple C, Farmani R, Fu G, Ward S, Butler D (2016) Global resilience analysis of water distribution systems. Water Res 106:383-393. doi.org/10.1016/j.watres.2016.10.011

Eidsvig UMK, Kristensen K, Vangelsten BV (2017) Assessing the risk posed by natural hazards to infrastructures. Nat Hazards Earth Syst Sci 17:481-504. doi:10.5194/nhess-17-481-2017.

EPA (2015) Systems Measures of Water Distribution System Resilience. EPA 600/R-14/383.

Fragiadakis M, Christodoulou SE, Vamvatsikos D (2013) Reliability Assessment of Urban Water Distribution Networks Under Seismic Loads. Water Resour Manage 27: 3739-3764. doi:10.1007/s11269-013-0378-0

Francis RA, Guikema SD, Henneman L. (2014) Bayesian Belief Networks for predicting drinking water distribution system pipe breaks. Reliab Eng Syst Saf 130:1-11. doi: 10.1016/j.ress.2014.04.024. 
1 Gaudard L, Romerio F (2015) Natural hazard risk in the case of an emergency: the real options' 1

22 approach. Nat Hazards 75(1): 473-488. doi:10.1007/s11069-014-1330-1

Gonzalez-Redin J, Luque S, Poggio L, Smith R, Gimona A (2016) Spatial Bayesian belief networks as a planning decision tool for mapping ecosystem services trade-offs on forested landscapes. Environ Res 144:15-26. doi: 10.1016/j.envres.2015.11.009.

John A, Yang Z, Riahi R, Wang J (2016) A risk assessment approach to improve the resilience of a seaport system using Bayesian networks. Ocean Eng 111:136-147. doi: 10.1016/j.oceaneng.2015.10.048.

Johnson S, Low-Choy S, Mengersen K (2011) Integrating Bayesian Networks and Geographic Information Systems: Good Practice Examples. Integr Environ Assess Manag 8(3): 473-479. doi: 10.1002/ieam.262

Kabir G, Demissie G, Sadiq R, Tesfamariam S (2015) Integrating failure prediction models for water mains: Bayesian belief network based data fusion. Knowl Based Syst 85:159-169. doi. 10.1016/j.knosys.2015.05.002

Kabir G, Sadiq R, Tesfamariam S (2016) A fuzzy Bayesian belief network for safety assessment of oil and gas pipelines. Struct Infrastruct Eng 12(8):874-889. doi:10.1080/15732479.2015.1053093

Karimi HA, Houston BH (1996) Evaluating strategies for integrating environmental models with GIS: current trends and future needs. Comput Environ Urban Syst 20(6):413-425. doi: 10.1016/S0198-9715(97)00006-9

Kongar I, Esposito S, Giovinazzi S (2017) Post-earthquake assessment and management for infrastructure systems: learning from the Canterbury (New Zealand) and L'Aquila (Italy) earthquakes. Bull Earthq Eng 15(2): 589-620. doi: 10.1007/s10518-015-9761-y 
Landuyt D, Van der Biest K Broekx S, Staes J Meire P, Goethals PLM (2015) A GIS plug-in for Bayesian belief networks: Towards a transparent software framework to assess and visualise uncertainties in ecosystem service mapping. Environ Model Softw 71:30-38. doi: 10.1016/j.envsoft.2015.05.002.

Liu R, Chen Y, Wu J, Gao L, Barrett D, Xu T, Li L, Huang C, Yu J (2016) Assessing spatial likelihood of flooding hazard using naive Bayes and GIS: a case study in Bowen Basin, Australia. Stoch Environ Res Risk Assess 30(6):1575-1590. doi: 10.1007/s00477-015-1198-y

Mala-Jetmarova H, Sultanova N, Savic D (2017) Lost in optimisation of water distribution systems? A literature review of system operation, Environ Model Softw 93:209-254. doi:10.1016/j.envsoft.2017.02.009.

Marcot BG (2012) Metrics for evaluating performance and uncertainty of Bayesian network models. Ecol Model 230:50- 62. doi: 10.1016/j.ecolmodel.2012.01.013

McCormick S (2016) New tools for emergency managers: an assessment of obstacles to use and implementation. Disasters 40(2): 207-225. doi: 10.1111/disa.12141.

Molina JL, Farmani R, Bromley J (2011) Aquifers management through evolutionary bayesian networks:the Altiplano case study (SE Spain). Water Resour Manag 25(14):3883-3909. doi:10.1007/s11269-011-9893-Z

Molina JL, Zazo S, Rodríguez-Gonzálvez P, González-Aguilera D (2016) Innovative Analysis of Runoff Temporal Behavior through Bayesian Networks. Water 8(11), 484. doi:10.3390/w8110484

Pagano A, Giordano R, Portoghese I, Fratino U, Vurro M (2014a) A Bayesian vulnerability assessment tool for drinking water mains under extreme events. Nat Hazards 74(3):2193-2227. doi: $10.1007 / \mathrm{s} 11069-014-1302-5$ 
1 Pagano A, Giordano R, Portoghese I, Vurro M, Fratino U (2014b) Emergency Management of

1

Drinking Water Infrastructures Based on a Bayesian Decision Support System. Vulnerability, Uncertainty, and Risk: Quantification, Mitigation, and Management - Proceedings of the 2nd International Conference on Vulnerability and Risk Analysis and Management, ICVRAM 2014 and the 6th International Symposium on Uncertainty Modeling and Analysis, ISUMA 2014, pp. 20122021.

Pagano A, Pluchinotta I, Giordano R, Vurro M (2017) Drinking water supply in resilient cities: notes from L'Aquila earthquake case study. Sustain Cities Soc 28:435-449. doi: 10.1016/j.scs.2016.09.005.

Pearl J (1988) Probabilistic Reasoning in Intelligent Systems, Morgan Kaufmann, San Francisco

Perng SY, Buscher M (2015) Uncertainty and Transparency: Augmenting Modelling and Prediction for Crisis Response, Proceedings of the ISCRAM 2015 Conference, Kristiansand, May 24-27, Palen, Büscher, Comes \& Hughes eds.

Phan TD, Smart JCR, Capon SJ, Hadwen WL, Sahin O (2016) Applications of Bayesian belief networks in water resource management: A systematic review. Environ Model Softw 85:98-111. doi: 10.1016/j.envsoft.2016.08.006

Sobradelo R, Martı J, Kilburn C, Lopez C (2015) Probabilistic approach to decision-making under uncertainty during volcanic crises: retrospective application to the El Hierro (Spain) 2011 volcanic crisis. Nat Hazards 76:979-998. doi: 10.1007/s11069-014-1530-8

Tanyimboh TT (2017) Informational Entropy: a Failure Tolerance and Reliability Surrogate for Water Distribution Networks. Water Resour Manage 31:3189-3204. doi:10.1007/s11269-017-16848

Tateosian L (2015) Python For ArcGIS. Springer. doi: 10.1007/978-3-319-18398-5 
1 Uusitalo L (2007) Advantages and challenges of Bayesian networks in environmental modeling. Ecol 1

22 Model 203(3-4):312-318. doi:10.1016/j.ecolmodel.2006.11.033

Uusitalo L, Lehikoinen A, Helle I, Myrberg K (2015) An overview of methods to evaluate uncertainty of deterministic models in decision support. Environ Model Softw 63:24-31. doi: 10.1016/j.envsoft.2014.09.017

van der Keur P, van Bers C, Henriksen HJ, Nibanupudi HK, Yadav S, Wijaya R, Subiyono A, Mukerjee N, Hausmann HJ, Hare M, van Scheltinga CT, Pearn G, Jaspers F (2016) Identification and analysis of uncertainty in disaster risk reduction and climate change adaptation in South and Southeast Asia, Int J Disaster Risk Reduct 16: 208-214. doi:10.1016/j.ijdrr.2016.03.002

Wu J, Zhou R, Xu S, Wu Z (2017) Probabilistic analysis of natural gas pipeline network accident based on Bayesian network, Journal of Loss Prevention in the Process Industries, 46:126-136. doi:10.1016/j.jlp.2017.01.025.

Zhao X, Cai H, Chen Z, Gong H, Feng Q (2016) Assessing urban lifeline systems immediately after seismic disaster based on emergency resilience. Struct Infrastruct Eng 12(12):1634-1649. doi: 10.1080/15732479.2016.1157609 


\begin{tabular}{|c|c|c|c|c|}
\hline Node & Mutual Info & Percent & Variance of Beliefs & Scenario \\
\hline Breaking Vulnerability & 1.3976 & 100 & 0.363296 & \\
\hline External stress level & 0.19371 & 13.9 & 0.044494 & \\
\hline Mechanical features & 0.09952 & 7.12 & 0.02237 & \\
\hline Physical vulnerability & 0.04676 & 3.35 & 0.01062 & \\
\hline Seismicity & 0.04403 & 3.15 & 0.010404 & $(1),(3)$ \\
\hline Existing instabilities & 0.02028 & 1.45 & 0.004848 & $(1),(3)$ \\
\hline Actual conditions & 0.01908 & 1.37 & 0.004305 & \\
\hline Soil mechanical characteristics & 0.01267 & 0.907 & 0.002837 & (3) \\
\hline Hydraulic efficiency & 0.01221 & 0.874 & 0.002945 & \\
\hline Safety level & 0.00808 & 0.578 & 0.001839 & \\
\hline Extra-maintenance & 0.0056 & 0.401 & 0.001275 & $(2),(3)$ \\
\hline OP/NP & 0.00312 & 0.223 & 0.000758 & (2) \\
\hline Dynamic loads & 0.00269 & 0.193 & 0.000649 & (1) \\
\hline Flexibility & 0.00212 & 0.152 & 0.000485 & \\
\hline Hydraulic variability & 0.00138 & 0.0991 & 0.000338 & \\
\hline Age/Design life & 0.00111 & 0.0797 & 0.000256 & (2) \\
\hline Joint extraction vulnerability & 0.00084 & 0.0598 & 0.000204 & \\
\hline Maintenance: performed/scheduled & 0.00077 & 0.0548 & 0.000175 & (2) \\
\hline Joint type & 0.00063 & 0.0452 & 0.000145 & (2) \\
\hline Diameter & 0.00059 & 0.0422 & 0.000137 & (2) \\
\hline Depth & 0.0004 & 0.0283 & $9.49 \mathrm{E}-05$ & (2) \\
\hline Joint frequency & 0.00014 & 0.0102 & $3.25 \mathrm{E}-05$ & (2) \\
\hline Corrosion vulnerability & 0.00004 & 0.00251 & 0.000008 & \\
\hline Pipe coating & 0.00003 & 0.00235 & 7.9E-06 & \\
\hline Cathodic protection & 0.00001 & 0.000767 & $2.6 \mathrm{E}-06$ & \\
\hline Thrust restraint & 0 & 0 & 0 & (2) \\
\hline
\end{tabular}




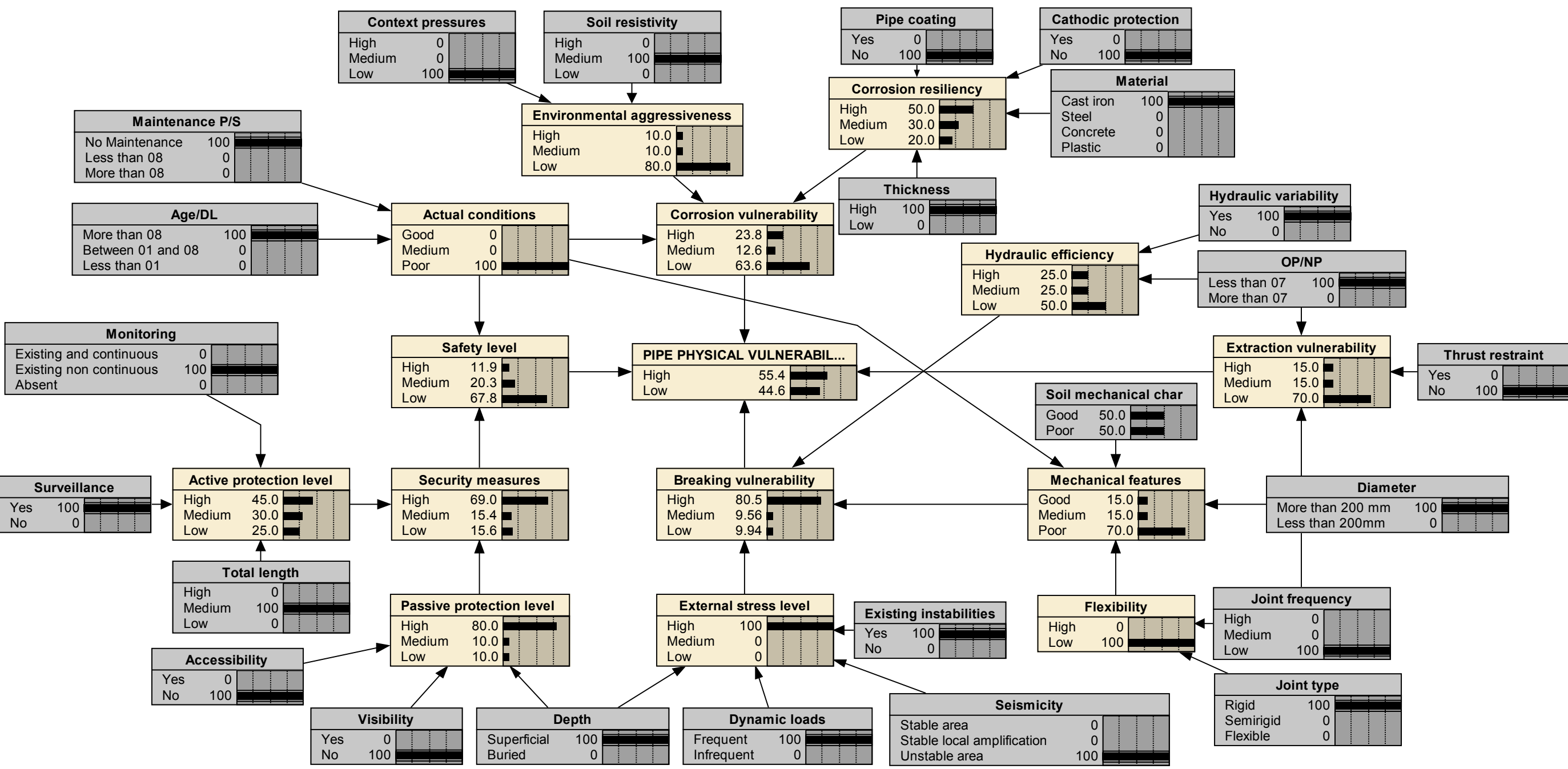




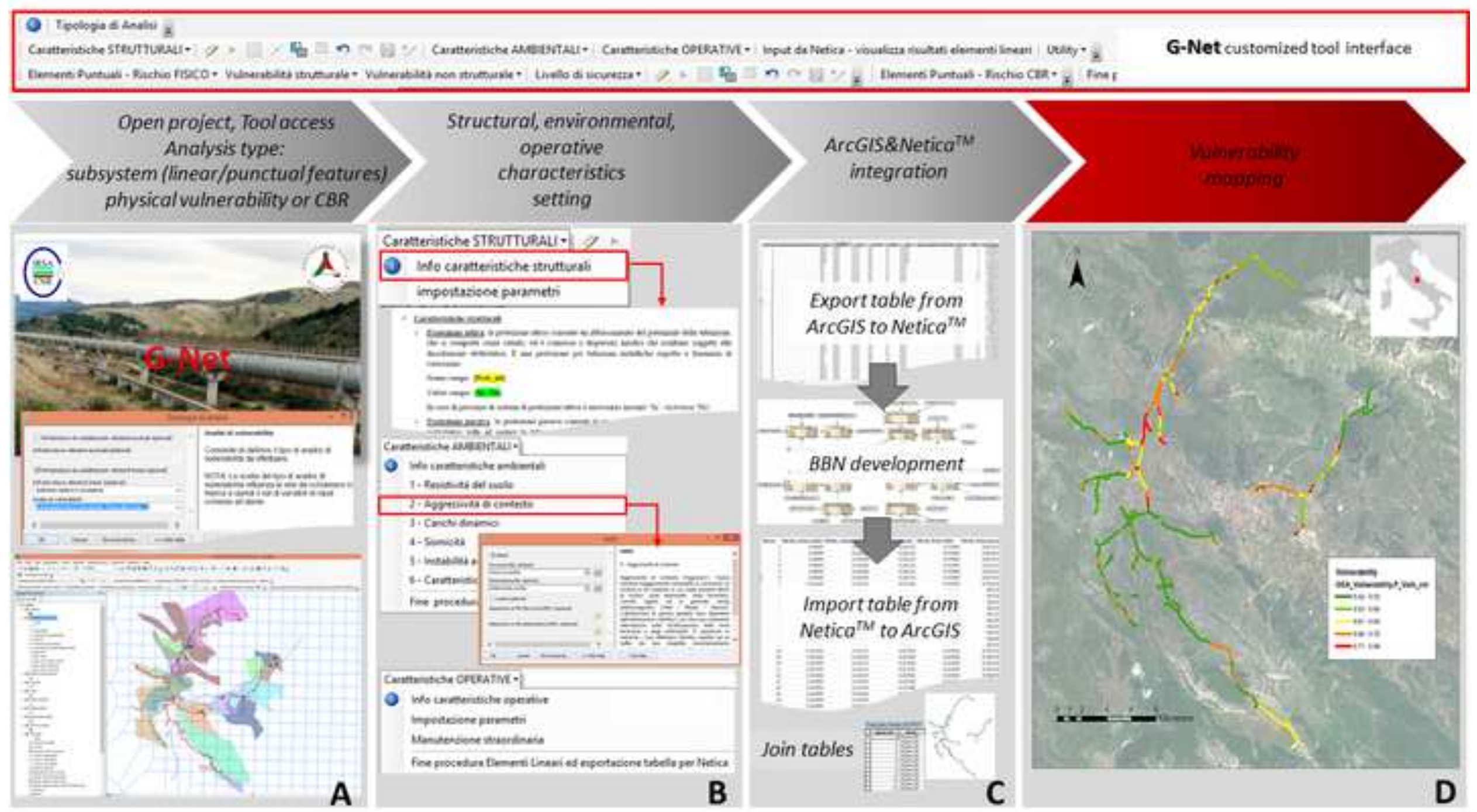




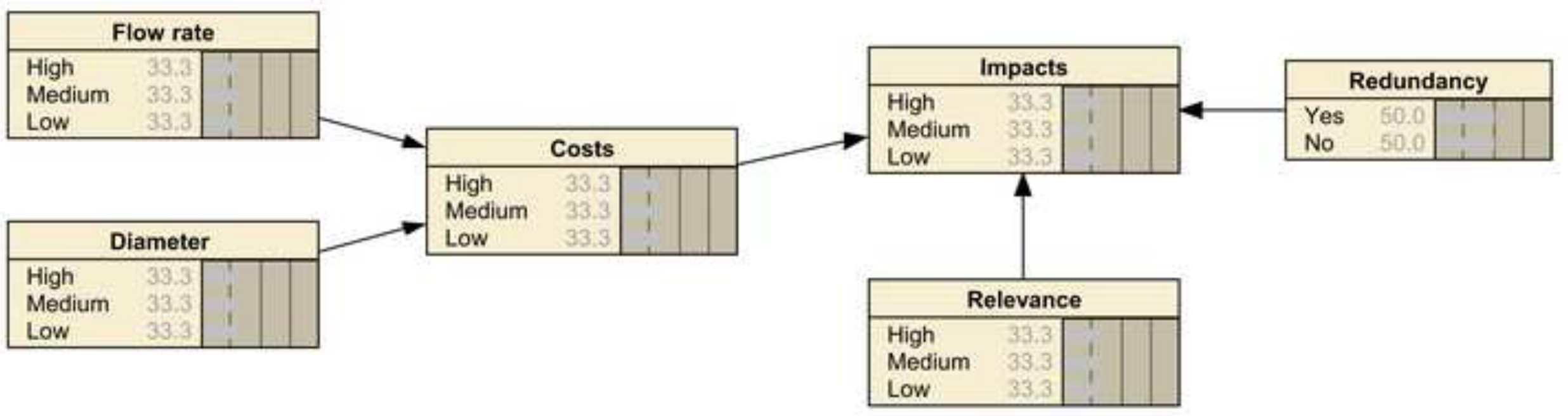




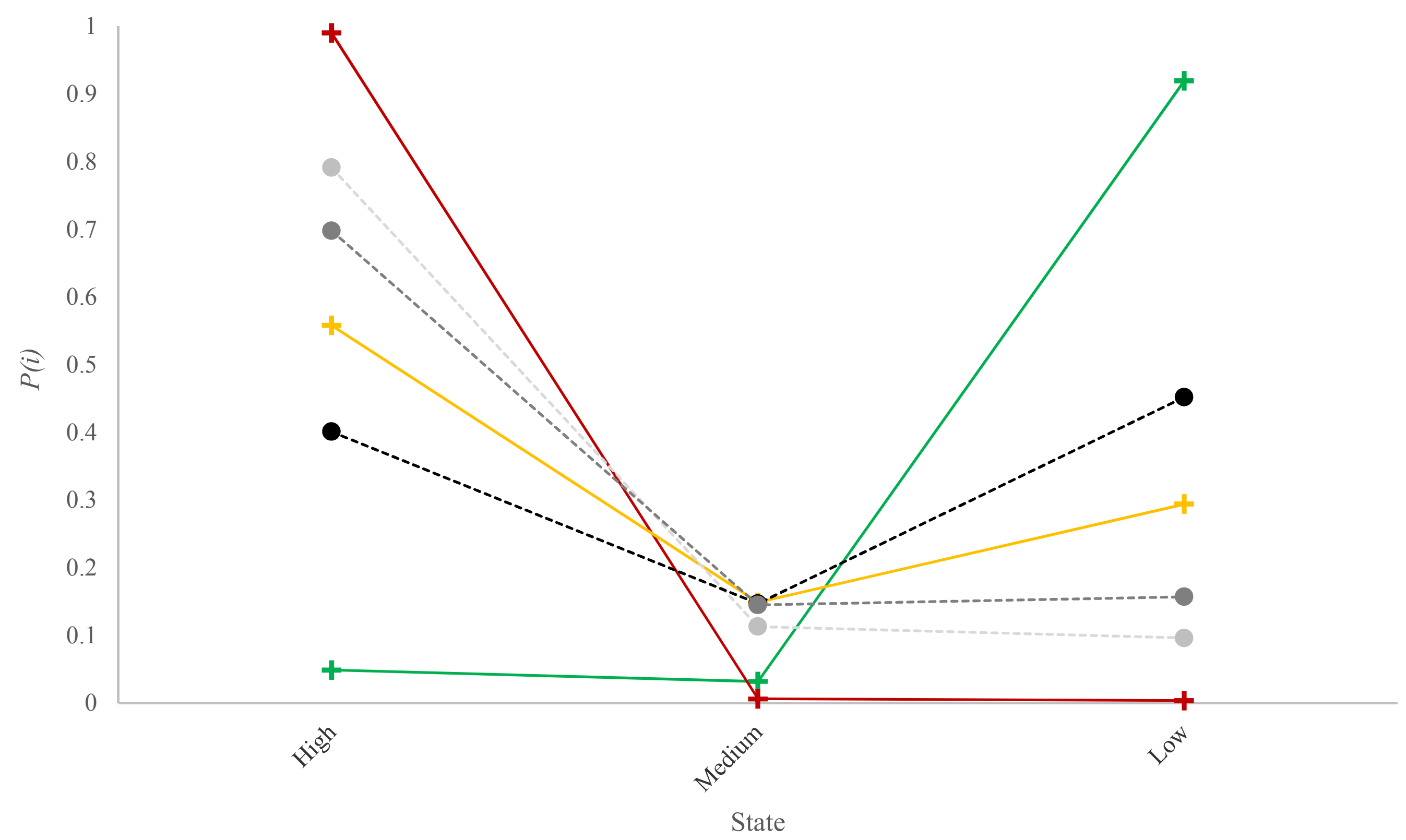




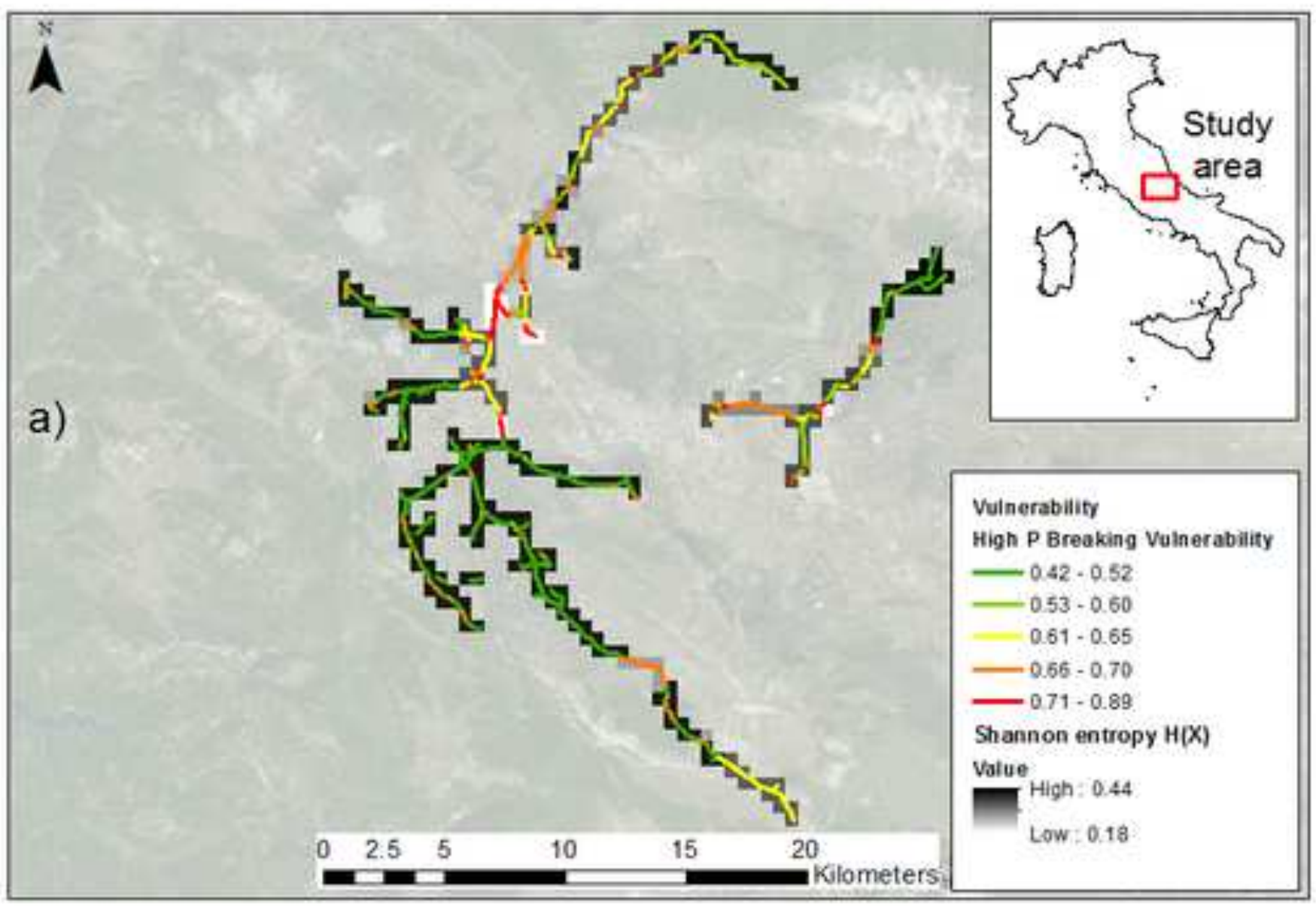

b)

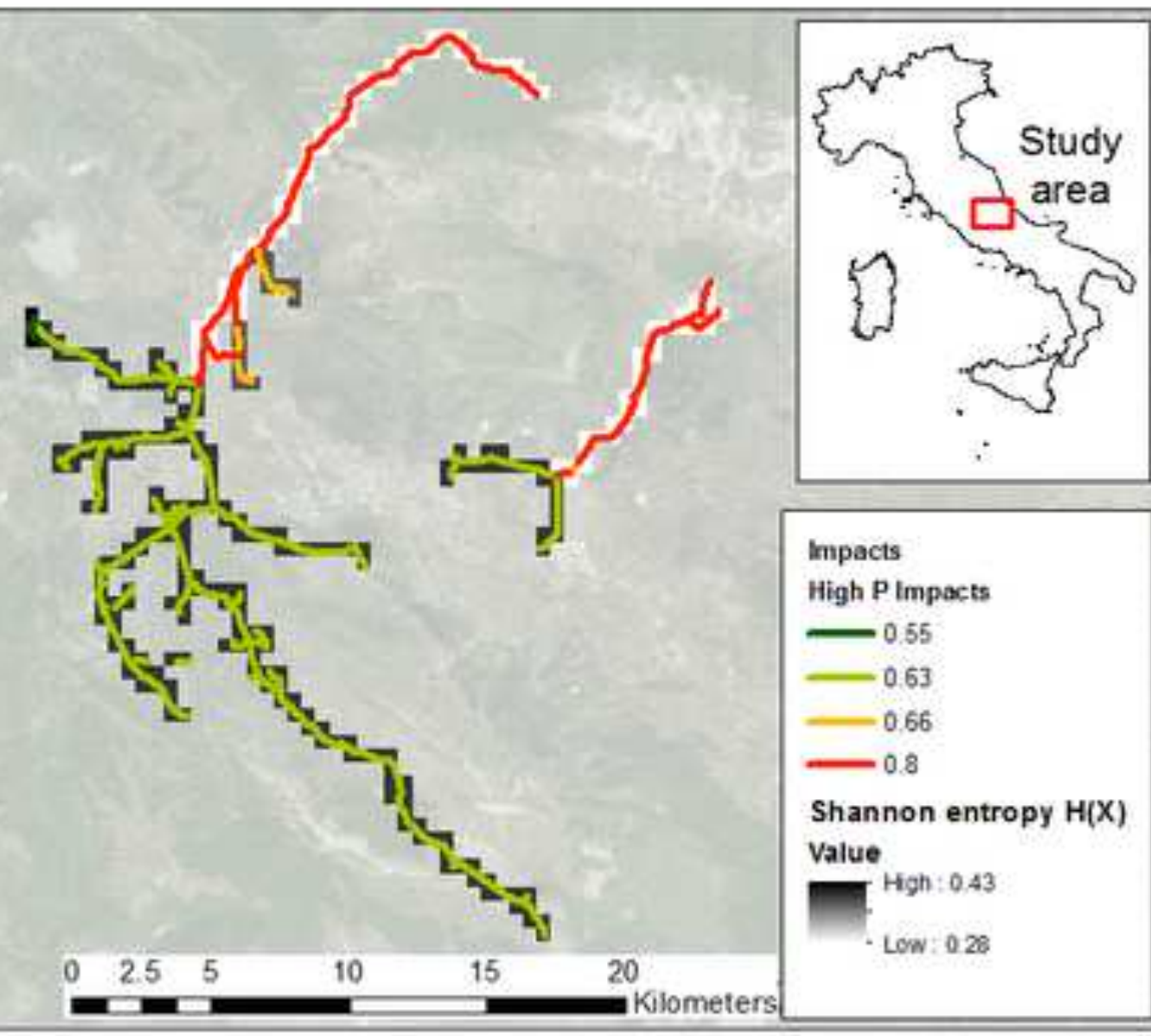




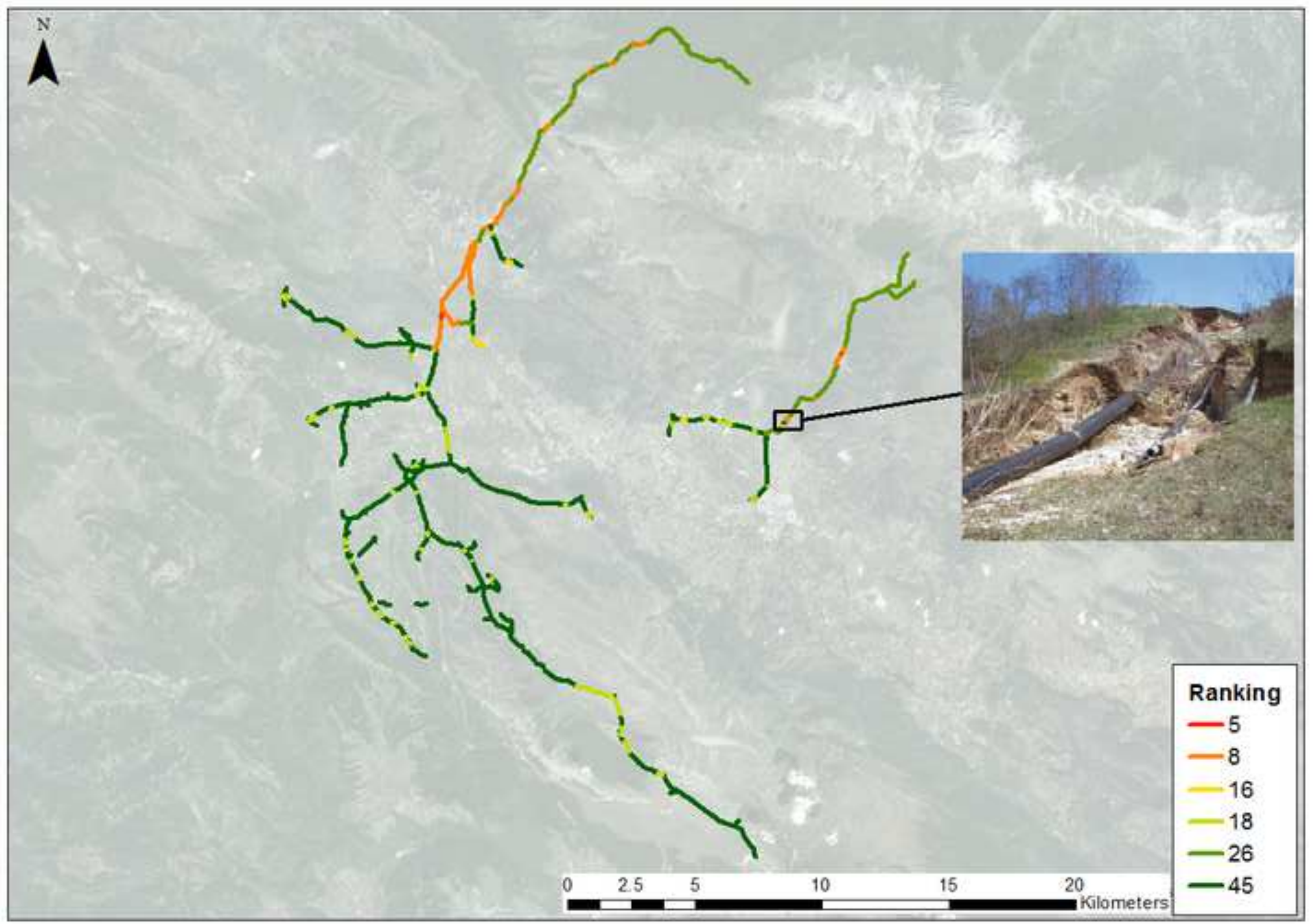




\section{Analysis and validation of the BBN-based vulnerability assessment tool}

2 The present section aims at providing additional details on the BBN-based vulnerability assessment 3 methodology, mainly focusing on a set of specific information related to model building and 4 validation.

5 The following Table S1 (from Pagano et al. 2014a) includes a detailed description of all the input 6 variables included in the BBN proposed in Fig. 1 of the paper. The meaning and the states of the 7 variables are included. It is worth to consider that mutual exclusivity is encoded via the states of 8 nodes, having particular attention in a proper identification of specific causal pathways (i.e. the 9 specific vulnerability mechanisms).

Table S1 Description of the input variables adopted, of their meaning and states

\begin{tabular}{|c|c|c|}
\hline Input variable & Meaning & States \\
\hline Material & $\begin{array}{l}\text { Different materials determine variable mechanical behaviors and show a } \\
\text { specific response to corrosion, breaking and deterioration phenomena. }\end{array}$ & $\begin{array}{ll}- & \text { Cast iron } \\
- & \text { Steel } \\
- & \text { Concrete } \\
- & \text { Plastic }\end{array}$ \\
\hline Thickness & A greater thickness accounts for greater resistance and corrosion resiliency. & $\begin{array}{ll}- & \text { High } \\
- & \text { Low }\end{array}$ \\
\hline Pipe coating & $\begin{array}{l}\text { Inner and outer pipe coatings guarantee optimal resistance to chemical } \\
\text { actions, deterioration and corrosion. }\end{array}$ & $\begin{array}{ll}- & \text { Yes } \\
- & \text { No }\end{array}$ \\
\hline Cathodic protection & $\begin{array}{l}\text { Active protection systems reduce pipe electrical potential limiting } \\
\text { corrosion. }\end{array}$ & $\begin{array}{ll}- & \text { Yes } \\
- & \text { No }\end{array}$ \\
\hline Thrust restraint & $\begin{array}{l}\text { The presence of thrust restraints balances specific forces (e.g. } \\
\text { hydrodynamic force in curves) }\end{array}$ & $\begin{array}{ll}- & \text { Yes } \\
- & \text { No }\end{array}$ \\
\hline Diameter & $\begin{array}{l}\text { Studies have shown that pipe breaks tend to reduce for pipes with greater } \\
\text { diameters. }\end{array}$ & $\begin{array}{l}->200 \mathrm{~mm} \\
-\quad<200 \mathrm{~mm}\end{array}$ \\
\hline Joint type & The flexibility of pipe joints conditions their response to external actions. & $\begin{array}{ll}- & \text { Rigid } \\
- & \text { Semi-rigid } \\
- & \text { Flexible } \\
\end{array}$ \\
\hline Joint frequency & The frequency of pipe joints conditions the overall flexibility of the system. & $\begin{array}{ll}- & \text { High } \\
- & \text { Medium } \\
- & \text { Low }\end{array}$ \\
\hline Depth & $\begin{array}{l}\text { Buried systems are less exposed to superficial events (e.g. floods) and often } \\
\text { not clearly visible. }\end{array}$ & $\begin{array}{ll}- & \text { Superficial } \\
- & \text { Buried }\end{array}$ \\
\hline Length & $\begin{array}{l}\text { The higher the length of the system, the lower the effectiveness of } \\
\text { monitoring activities. }\end{array}$ & $\begin{array}{ll}- & \text { High } \\
- & \text { Medium } \\
- & \text { Low }\end{array}$ \\
\hline $\begin{array}{l}\text { Soil mechanical } \\
\text { characteristics }\end{array}$ & $\begin{array}{l}\text { The mechanical properties of soil and backfill properties influence the } \\
\text { system's response to external actions. }\end{array}$ & $\begin{array}{ll}- & \text { Good } \\
- & \text { Poor }\end{array}$ \\
\hline Seismicity & $\begin{array}{l}\text { The expected external stress level is characterized also through the analysis } \\
\text { of the seismicity of the investigated area. }\end{array}$ & $\begin{array}{ll}- & \text { High } \\
- & \text { Medium } \\
- & \text { Low } \\
\end{array}$ \\
\hline Existing instabilities & $\begin{array}{l}\text { Increasing vulnerabilities are expected where local instabilities (e.g. faults } \\
\text { or landslides) already exist. }\end{array}$ & $\begin{array}{ll}- & \text { Yes } \\
- & \text { No }\end{array}$ \\
\hline Dynamic loads & $\begin{array}{l}\text { The higher the dynamic loads (e.g. traffic loads) the higher the system's } \\
\text { vulnerability. }\end{array}$ & $\begin{array}{ll}\text { - } & \text { Frequent } \\
\text { - } & \text { Absent }\end{array}$ \\
\hline External pressures & $\begin{array}{l}\text { Local aggressive conditions (e.g. proximity of electricity lines, external } \\
\text { currents) may increase vulnerability levels. }\end{array}$ & $\begin{array}{ll}- & \text { High } \\
- & \text { Medium } \\
- & \text { Low } \\
\end{array}$ \\
\hline Soil resistivity & $\begin{array}{l}\text { Soil resistivity summarizes a series of soil chemical, physical and } \\
\text { biological features determining the expected behavior in terms of } \\
\text { corrosion. }\end{array}$ & $\begin{array}{ll}- & \text { High } \\
- & \text { Medium } \\
- & \text { Low }\end{array}$ \\
\hline
\end{tabular}




\begin{tabular}{|c|c|c|}
\hline Hydraulic variability & $\begin{array}{l}\text { A water system is much more vulnerable if subjected to significant } \\
\text { variations in hydraulic conditions, particularly pressure. In the case of } \\
\text { water mains, the entity of hydrostatic pressure is considered. }\end{array}$ & $\begin{array}{ll}- & \text { High } \\
- & \text { Medium } \\
- & \text { Low }\end{array}$ \\
\hline $\begin{array}{l}\text { Operating Pressure / } \\
\text { Nominal Pressure }\end{array}$ & $\begin{array}{l}\text { A pipe is much more vulnerable if operating pressure is close to its nominal } \\
\text { pressure. }\end{array}$ & $\begin{array}{ll}- & \text { High }(0.66-1) \\
- & \text { Medium }(0.33-0.66) \\
- & \text { Low }(0-0.33)\end{array}$ \\
\hline Visibility & $\begin{array}{l}\text { Most hydraulic structures are hidden. Recognizable structures are more } \\
\text { exposed to sabotage and terrorist acts. }\end{array}$ & $\begin{array}{ll}- & \text { Yes } \\
- & \text { No }\end{array}$ \\
\hline Accessibility & $\begin{array}{l}\text { Accessible structures (without fences or walls) are more exposed to } \\
\text { sabotage and terrorist acts. }\end{array}$ & $\begin{array}{ll}- & \text { Yes } \\
- & \text { No }\end{array}$ \\
\hline Surveillance & $\begin{array}{l}\text { Surveillance by employees or monitoring systems reduces the risk of } \\
\text { intrusion and accelerates emergency responses. }\end{array}$ & $\begin{array}{ll}- & \text { Yes } \\
-\quad & \text { No }\end{array}$ \\
\hline Monitoring & $\begin{array}{l}\text { Qualitative and quantitative monitoring systems (both local and } \\
\text { centralized), especially if continuous, help in quickly detecting problems } \\
\text { and faults. }\end{array}$ & $\begin{array}{ll}- & \text { Existing and continuous } \\
- & \text { Existing non continuous } \\
\text { - } & \text { Absent }\end{array}$ \\
\hline Age / Design Life & $\begin{array}{l}\text { Failure probability follows the classical 'bathtub' curve: older systems are } \\
\text { less efficient and more subject to deterioration, newly completed ones may } \\
\text { be affected by construction faults. }\end{array}$ & $\begin{array}{ll}- & >0.8 \\
- & 0.1-0.8 \\
- & <0.1\end{array}$ \\
\hline $\begin{array}{l}\text { Maintenance: } \\
\text { Performed/Scheduled }\end{array}$ & $\begin{array}{l}\text { Regular maintenance contributes to improving pipe conditions and } \\
\text { response to external stresses. }\end{array}$ & $\begin{array}{ll}- & \text { Low } \\
- & \text { Medium } \\
- & \text { High }\end{array}$ \\
\hline Extra Maintenance & $\begin{array}{l}\text { Past unexpected maintenance activities denote vulnerable areas or } \\
\text { vulnerability conditions due to local factors. }\end{array}$ & $\begin{array}{ll}- & \text { Frequent } \\
- & \text { Absent }\end{array}$ \\
\hline
\end{tabular}

The variables included in the model (the total number of nodes is 40) were also topologically ordered. Given a DAG, the topological ordering of variables $\left(X_{1}, X_{2}, \ldots, X_{n}\right)$ is an ordering in which parents are ordered before the children. The topological order (one of the possible topological orders) of the elements of the network is: (External pressures, Soil resistivity, Material, Pipe Coating, Cathodic protection, Thickness, Hydraulic variability, Operating pressure/Nominal pressure, Thrust restraint, Soil mechanical characteristics, Diameter, Joint Frequency, Joint type, Seismicity, Existing Instabilities, Dynamic loads, Depth, Visibility, Accessibility, Surveillance, Length, Monitoring, Extra maintenance, Age/Design life, Maintenance performed/scheduled; Environmental aggressiveness, Corrosion resiliency, Hydraulic efficiency, Joint extraction vulnerability, Mechanical features, External stress level, 'Passive' protection level, 'Active' protection level, Actual conditions; Protection level, Corrosion vulnerability, Breaking vulnerability, Safety level; Physical vulnerability).

D-Separation can be considered in order to analyze independence of nodes. Particularly, according to the D-separation rule, $\mathrm{A}$ is d-separated from $\mathrm{B}$ by $\mathrm{C}$ if all the paths between sets $\mathrm{A}$ and $\mathrm{B}$ are blocked by elements of $\mathrm{C}$. Such rule enables to quickly determine whether a finding at one node can possibly change the beliefs at another by only looking at the link structure of a Bayes net. Equivalently, DConnected nodes can be also identified, i.e. the nodes whose beliefs could change if findings were obtained for a currently selected node, based on the graph connectivity (or vice-versa). The following table S2 summarizes, for each node of the BBN, the set of D-Connected nodes (the complementary sub-set will be D-Separated).

Table S2. D-connected nodes

\begin{tabular}{ll}
\multicolumn{1}{c}{ Node } & \multicolumn{1}{c}{ D-connected nodes } \\
\hline External pressures & Environmental aggressiveness, Corrosion vulnerability, Physical vulnerability \\
\hline Soil resistivity & Environmental aggressiveness, Corrosion vulnerability, Physical vulnerability \\
\hline Material & Corrosion resiliency, Corrosion vulnerability, Physical vulnerability \\
\hline
\end{tabular}




\begin{tabular}{|c|c|}
\hline Pipe Coating & Corrosion resiliency, Corrosion vulnerability, Physical vulnerability \\
\hline Cathodic protection & Corrosion resiliency, Corrosion vulnerability, Physical vulnerability \\
\hline Thickness & Corrosion resiliency, Corrosion vulnerability, Physical vulnerability \\
\hline Hydraulic variability & Hydraulic efficiency, Breaking vulnerability, Physical vulnerability \\
\hline $\begin{array}{l}\text { Operating pressure/nominal } \\
\text { pressure }\end{array}$ & $\begin{array}{l}\text { Hydraulic efficiency, Joint extraction vulnerability, Breaking vulnerability, } \\
\text { Physical vulnerability }\end{array}$ \\
\hline Thrust restraint & Joint extraction vulnerability, Physical vulnerability \\
\hline Soil mechanical characteristics & Mechanical features, Breaking vulnerability, Physical vulnerability \\
\hline Diameter & Mechanical features, Breaking vulnerability, Physical vulnerability \\
\hline Joint frequency & $\begin{array}{l}\text { Flexibility, Mechanical features, Joint extraction vulnerability, Breaking } \\
\text { vulnerability, Physical vulnerability }\end{array}$ \\
\hline Joint type & Flexibility, Mechanical features, Breaking vulnerability, Physical vulnerability \\
\hline Seismicity & External stress level, Breaking vulnerability, Physical vulnerability \\
\hline Existing instabilities & External stress level, Breaking vulnerability, Physical vulnerability \\
\hline Dynamic loads & External stress level, Breaking vulnerability, Physical vulnerability \\
\hline Depth & $\begin{array}{l}\text { External stress level, 'Passive' protection level, Protection level, Safety level, } \\
\text { Breaking vulnerability, Physical vulnerability }\end{array}$ \\
\hline Visibility & 'Passive' protection level, Protection level, Safety level, Physical vulnerability \\
\hline Accessibility & 'Passive' protection level, Protection level, Safety level, Physical vulnerability \\
\hline Surveillance & 'Active' protection level, Protection level, Safety level, Physical vulnerability \\
\hline Length & 'Active' protection level, Protection level, Safety level, Physical vulnerability \\
\hline Monitoring & 'Active' protection level, Protection level, Safety level, Physical vulnerability \\
\hline Extra maintenance & $\begin{array}{l}\text { Actual conditions, Safety level, Corrosion vulnerability, Mechanical features, } \\
\text { Breaking vulnerability, Physical vulnerability }\end{array}$ \\
\hline Age/Design life & $\begin{array}{l}\text { Actual conditions, Safety level, Corrosion vulnerability, Mechanical features, } \\
\text { Breaking vulnerability, Physical vulnerability }\end{array}$ \\
\hline $\begin{array}{l}\text { Maintenance: } \\
\text { performed/scheduled }\end{array}$ & $\begin{array}{l}\text { Actual conditions, Safety level, Corrosion vulnerability, Mechanical features, } \\
\text { Breaking vulnerability, Physical vulnerability }\end{array}$ \\
\hline Environmental aggressiveness & $\begin{array}{l}\text { Soil resistivity, External pressures, Corrosion vulnerability, Physical } \\
\text { vulnerability }\end{array}$ \\
\hline Corrosion resiliency & $\begin{array}{l}\text { Material, Pipe coating, Cathodic protection, Thickness, Corrosion vulnerability, } \\
\text { Physical vulnerability }\end{array}$ \\
\hline Hydraulic efficiency & $\begin{array}{l}\text { Hydraulic variability, Operating pressure/Nominal pressure, Joint extraction } \\
\text { vulnerability, Breaking vulnerability, Physical vulnerability }\end{array}$ \\
\hline Joint extraction vulnerability & $\begin{array}{l}\text { Hydraulic efficiency, Operating pressure/Nominal pressure, Thrust restraint, } \\
\text { Joint frequency, Flexibility, Mechanical features, Breaking vulnerability, } \\
\text { Physical vulnerability }\end{array}$ \\
\hline Mechanical features & $\begin{array}{l}\text { Joint extraction vulnerability, Diameter, Joint frequency, Diameter, Joint type, } \\
\text { Flexibility, Soil mechanical characteristics, Breaking vulnerability, Physical } \\
\text { vulnerability, Corrosion vulnerability, Safety level, Actual conditions, Extra- } \\
\text { maintenance, Age/Design life, Maintenance: performed/scheduled. }\end{array}$ \\
\hline Flexibility & $\begin{array}{l}\text { Joint type, Joint frequency, Joint extraction vulnerability, Mechanical features, } \\
\text { Breaking vulnerability, Physical vulnerability }\end{array}$ \\
\hline External stress level & $\begin{array}{l}\text { Seismicity, Existing instabilities, Dynamic loads, Depth, 'Passive' protection } \\
\text { level, Protection level, Safety level, Breaking vulnerability, Physical } \\
\text { vulnerability. }\end{array}$ \\
\hline 'Passive' protection level & $\begin{array}{l}\text { Accessibility, Visibility, Depth, Protection level, Safety level, External stress } \\
\text { level, Breaking vulnerability, Physical vulnerability }\end{array}$ \\
\hline 'Active' protection level & $\begin{array}{l}\text { Surveillance, Length, Monitoring, Protection level, Safety level, Physical } \\
\text { vulnerability }\end{array}$ \\
\hline Actual conditions & $\begin{array}{l}\text { Extra-maintenance, Age/Design life, Maintenance: performed/scheduled, } \\
\text { Corrosion vulnerability, Breaking vulnerability, Mechanical features, Physical } \\
\text { vulnerability }\end{array}$ \\
\hline Corrosion vulnerability & $\begin{array}{l}\text { Extra maintenance, Age/Design life, External pressure, Maintenance: } \\
\text { performed/scheduled, Soil resistivity, Material, Pipe coating, Cathodic } \\
\text { protection, Thickness, Corrosion resiliency, Environmental aggressiveness, } \\
\text { Actual conditions, Safety level, Mechanical features, Breaking vulnerability, } \\
\text { Physical vulnerability }\end{array}$ \\
\hline
\end{tabular}


Protection level

Safety level
Length, Monitoring, Surveillance, 'Active' protection level, Accessibility, Visibility, Depth, 'Passive' protection level, External stress level, Protection level, Safety level, Breaking vulnerability, Physical vulnerability

Length, Monitoring, Surveillance, 'Active' protection level, Accessibility, Visibility, Depth, 'Passive' protection level, External stress level, Protection level, Safety level, Breaking vulnerability, Mechanical features, Safety level, Extra-maintenance, Age/Design life, Maintenance: performed/scheduled, Corrosion vulnerability, Physical vulnerability

Extra-maintenance, Age/Design life, Maintenance: performed/scheduled, Actual conditions, Corrosion vulnerability, Hydraulic variability, Hydraulic efficiency, Operating pressure/Nominal pressure, Joint extraction vulnerability, Diameter, Soil mechanical characteristics, Mechanical features, Flexibility, Jint frequency, Joint type, Seismicity, Existing instabilities, Dynamic loads, Depth, External stress level, 'Passive' protection level, Protection level, Safety level, Breaking vulnerability, Physical vulnerability

Physical vulnerability

In the following Table S3, the junction tree of the vulnerability assessment BBN is included. A junction tree is an internal structure that Netica uses for belief updating. Netica compiles a Bayes net or decision net into a junction tree for efficiency. The junction tree $T$ of triangulated net $G$ is a tree with the cliques of $\mathrm{G}$ as nodes, such that for every node $\mathrm{N}$ of $\mathrm{G}$, if we remove from $\mathrm{T}$ all cliques not containing $\mathrm{N}$, the remaining subtree remains connected. In other words, any two cliques containing $\mathrm{N}$ are either adjacent in $\mathrm{T}$ or connected by a path made entirely of cliques that contain $\mathrm{N}$.

Table S3. Junction tree

\begin{tabular}{|c|c|c|c|}
\hline Clique & [Joined To] & Size & Member nodes (* means home) \\
\hline 0 & {$\left[\begin{array}{ll}0 & 15\end{array}\right]$} & 54 & Protection level, Depth, *Safety level, Actual conditions \\
\hline 1 & {$\left[\begin{array}{lll}0 & 2 & 14\end{array}\right]$} & 54 & $\begin{array}{l}\text { Depth, Safety level, External stress level, Breaking vulnerability, } \\
\text { Actual conditions }\end{array}$ \\
\hline 2 & {$\left[\begin{array}{lll}1 & 3 & 5\end{array}\right]$} & 243 & $\begin{array}{l}\text { Safety level, External stress level, Actual conditions, Breaking } \\
\text { vulnerability, Joint extraction vulnerability }\end{array}$ \\
\hline 3 & {$\left[\begin{array}{lll}2 & 4 & 13\end{array}\right]$} & 243 & $\begin{array}{l}\text { Corrosion vulnerability, Safety level, Actual conditions, Breaking } \\
\text { vulnerability, Joint extraction vulnerability }\end{array}$ \\
\hline 4 & [3] & 162 & $\begin{array}{l}\text { *Physical vulnerability, Corrosion vulnerability, Safety level, } \\
\text { Breaking vulnerability, Joint extraction vulnerability }\end{array}$ \\
\hline 5 & [2 6] & 729 & $\begin{array}{l}\text { External stress level, Actual conditions, Mechanical features, } \\
\text { Hydraulic efficiency, *Breaking vulnerability, Joint extraction } \\
\text { vulnerability }\end{array}$ \\
\hline 6 & {$\left[\begin{array}{lll}5 & 7 & 8\end{array}\right]$} & 162 & $\begin{array}{l}\text { Flexibility, Actual conditions, Mechanical features, Hydraulic } \\
\text { efficiency, Joint extraction vulnerability }\end{array}$ \\
\hline 7 & {$[6]$} & 72 & $\begin{array}{l}\text { *Mechanical features, *Diameter, Flexibility, Actual conditions, } \\
\text { *Mechanical features }\end{array}$ \\
\hline 8 & {$\left[\begin{array}{lll}6 & 9 & 12\end{array}\right]$} & 54 & $\begin{array}{l}\text { Operating pressure/Nominal pressure, Hydraulic efficiency, Joint } \\
\text { extraction vulnerability }\end{array}$ \\
\hline 9 & {$\left[\begin{array}{llll}8 & 10 & 1\end{array}\right]$} & 54 & $\begin{array}{l}\text { Joint frequency, Operating pressure/Nominal pressure, Flexibility, } \\
\text { Joint extraction vulnerability }\end{array}$ \\
\hline 10 & [9] & 54 & $\begin{array}{l}\text { *Thrust restraint, Joint frequency, Operating pressure/Nominal } \\
\text { pressure, *Joint extraction vulnerability }\end{array}$ \\
\hline 11 & [9] & 18 & *Joint type, $*$ Joint frequency, *Flexibility \\
\hline 12 & [8] & 27 & $\begin{array}{l}\text { *Hydraulic variability, * Operating pressure/Nominal pressure, } \\
* \text { Hydraulic efficiency }\end{array}$ \\
\hline 13 & {$\left[\begin{array}{llll}3 & 18 & 19 & 20\end{array}\right]$} & 81 & $\begin{array}{l}\text { Environmental aggressiveness, Corrosion resiliency, *Corrosion } \\
\text { vulnerability, Actual conditions }\end{array}$ \\
\hline 14 & {$[1]$} & 72 & $\begin{array}{l}\text { *Existing instabilities, *Seismicity, *Dynamic loads, Depth, } \\
\text { *External stress level }\end{array}$ \\
\hline 15 & {$\left[\begin{array}{llll}0 & 16 & 17\end{array}\right]$} & 54 & $\begin{array}{l}\text { 'Passive' protection level, 'Active' protection level, *Protection } \\
\text { level, Depth }\end{array}$ \\
\hline
\end{tabular}




\begin{tabular}{|l|l|l|l|}
\hline 16 & {$[15]$} & 24 & *Visibility, *Accessibility, *'Passive' protection level, *Depth \\
\hline 17 & {$[15]$} & 54 & *Monitoring, *Surveillance, *Length, *'Active' protection level \\
\hline 18 & {$[13]$} & 96 & $\begin{array}{l}\text { *Material, *Pipe coating, *Cathodic protection, *Thickness, } \\
\text { *Corrosion resiliency }\end{array}$ \\
\hline 19 & {$[13]$} & 27 & $\begin{array}{l}\text { *External pressures, *Soil resistivity, *Environmental } \\
\text { aggressiveness }\end{array}$ \\
\hline 20 & {$[13]$} & 54 & $\begin{array}{l}\text { *Extra maintenance, *Age/Design life, *Maintenance: } \\
\text { performed/scheduled, *Actual conditions }\end{array}$ \\
\hline
\end{tabular}


1 Dealing with uncertainty in decision-making for drinking water supply systems exposed to

3 Alessandro Pagano ${ }^{1, *}$, Irene Pluchinotta ${ }^{2}$, Raffaele Giordano ${ }^{1}$, Anna Bruna Petrangeli ${ }^{1}$, Umberto

4 Fratino $^{3}$ and Michele Vurro ${ }^{1}$

51 Water Research Institute - National Research Council (IRSA-CNR)

6 alessandro.pagano@ba.irsa.cnr.it; petrangeli@irsa.cnr.it; raffaele.giordano@cnr.it;

7 michele.vurro@ba.irsa.cnr.it

8

92 LAMSADE - CNRS, Univ. Paris-Dauphine, PSL Research Univ.

10 irene.pluchinotta@dauphine.fr

113 DICATECh, Politecnico di Bari

12 umberto.fratino@poliba.it

13 * Correspondence: alessandro.pagano@ba.irsa.cnr.it; Tel.: +39-080-5820506 


\section{Abstract}

2 The availability and the quality of drinking water are key requirements for the well-being and the safety of a community, both in ordinary conditions and in case of disasters. Providing safe drinking

4 water in emergency contributes to limit the intensity and the duration of crises, and is thus one of the 5 main concerns for decision-makers, who-must. In such cases, decision makers have to-operate under 6 significant uncertainty-due to the incomplete and limited set of information available. The present

7 work proposes a Decision Support System for the emergency management of drinking water supply

8 systems, which is built integrating: i) a vulnerability assessment model based on Bayesian Belief

9 Networks; ii) with the related an-uncertainty assessment model; iii) a model for impact, and related 10 uncertainty assessment, based on Bayesian Belief Networks. The results of these models are jointly 11 analyzed, providing decision-makers with a ranking of the priority of intervention. A GIS interface $12(\mathrm{G}-\mathrm{Net})$ is developed to manage both input spatial information $\overline{\bar{z}}_{\overline{2}}$ and results. The methodology is 13 implemented in L'Aquila case study, which is particularly relevant in the recent history of disasters. 14 discussing Fthe potentialities associated to the use of Bayesian Networks to suppert decision 15 makersthe tool dealing with information and data uncertainty, are discussed.

Keywords: Emergency management; Drinking water supply systems; Bayesian Belief Networks; Uncertainty Analysis; Decision Support System 
Lifeline systems consist of a set of interconnected infrastructures (e.g. water, gas, electricity, communication, transportation systems) supporting the provision of critical services and contributing

4 to guarantee the quality of life for citizens (Zhao et al. 2016). Since mModern societies highly rely 5 on infrastructures, which provide critical services and guarantee the quality of life for citizens (Zhao

6 et al. 2016). Nevertheless $T$, the the eurrentincrease in both frequency and intensity of extreme events

7 contributes to create additional challenges to the infrastructure providers eperating in the aftermath

8 of high-impacts occurrences-(Eidsvig et al. 2017). Among all lifelinesParticularly, water supply

9 systems-infrastructures are essential for health, sanitary and economic reasons and, consequently, there is high pressure on water organizations to provide customers with a continual and efficient water supply, under specific delivery requirements and operational constraints (Bagheri et al. 2010,-MalaJetmarova et al. 2017).

Several approaches are mentioned in the scientific and grey literature aiming atavailable for protecting water supply infrastructures from a wide variety of stresses, either supporting system performances assessment in case of extreme events (e.g. EPA 2015) or driving the selection of suitable actions for vulnerabilities mitigation (Fragiadakis et al. 2013, Paganoet al. 2014a). Methods to assess the performances of infrastructural-systems under stress-typically vary with the type of system, the aim or of the specific phaseanalysis of the analysis (e.g. planning or emergency management), and the available information. Probabilistic modelling, statistical analyses of past events, empirical approaches, system dynamics based approaches, agent based approaches are mentioned in the literature (EPA 2015, Eidsvig et al. 2017).A broad classification is generally-into qualitative, semi-quantitative and quantitative approaches (Pagano et al. 2014aa; Eidsvig et al. 2017). Quantitative tools require detailed data and a higher computational burden, but generally provide highly-reliable numerical outcomes for decision-makingmakers, typieally using numerical values and detailed analyses of critical scenarios (e.g. Fragiadakis et al 2013, Diao et al. 2016). Qualitative 
1 approaches support ranking risk levels, screening scenarios-and identifying critical scenarios-ones

2 (Eidsvig et al. 2017), based on the use of words or classes (e.g. 'high', 'medium', 'low'). The class

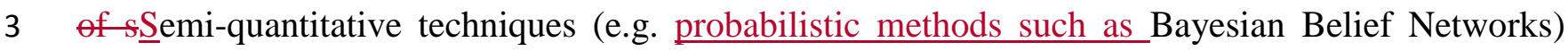
4 guarantees a compromise between the-such main features of the two-classes-of tools and data 5 requirement.

6 One of the most challenging tasks in allthese methods is uncertainty management, a key aspect als $\Theta$ to be incorporated in water supply systems management (Beh et al. 2017).

8 Uncertainty represents the lack of exact knowledge, regardless of its causes (Refsgaard et al. 9 2007).which is inherently Ffirstlyirst of all, uncertainty is-associated to water supply systems planning, design and eperationoperation, due_e.g. to structural characteristics and hydraulic capacity, variable demand and random fluctuations service level-((Malm et al. 2015, Tanyimboh 2017). Secondly-Specifically, particularly in emergency conditions, besides the uncertainties related to their emergency onset, nature and evolution (Perng and Buscher 2015), -) as well as the difficulty in collecting reliable data, model limitations, and the ambiguity in the understanding of specific phenomena-imply limitations in the capability to describe a given infrastructural system, and to forecast its behavioral evolution should be properly considered-during the emergency. This-These

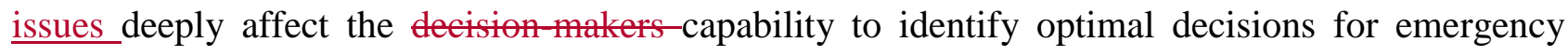
management (Pagano et al. 2014b, Gaudard and Romerio 2015). Several schelars highlighted the need to eEnhanceing the understanding of the uncertainty uncertainties could support in order to developing a realistierepresentative picture of the current knowledge and its potential deficiencies, and to avoid overconfidence in quantitative data and marginalization of non-quantifiable information (Uusitalo et al. 2015, Sword-Daniels et al. 2016,-van der Keur et al. 2016). Bayesian Belief Networks_(BBNs) have shown several useful features to support decision-making under uncertainty for water supply systems (Molina et al. 2011). FirstlyParticularly, BBNs allow the integration of various types of information,-(e.g. analytical models, expert knowledge, literature and 
1 historical_data),_combining qualitative and quantitative aspects (Giordano et al. 2015, Gonzalez-

2 Redin et al. 2016, Phan et al. 2016) that can be combined also with new variables and knowledge

3 (Landuyt et al. 2013, Genzalez Redin et al. 2016). and-. They Secondly, they support reasoning from

4 uncertain evidence to uncertain conclusion (John et al. 2016), treating both . The uncertainties (data

5 and model uncertainty, model uncertainty or both) are explicitly treated and included in BBNs by

6 propagating them throughout the network up to the final node (Uusitalo 2007, Marcot 2012, Uusitalo

7 et al. 2015, Gonzalez-Redin et al. 2016).More specifically, they can easily handle missing or little

8 data, and typically yield good prediction. Furthermore, BBNs also represent a valuable tool for

9 decision-makers, since costs and risks associated to different management strategies can be easily

assessed (Uusitalo, 2007; Mohajerani et al. 2017).

Within this framework, the present work describes the development of a Decision Support System (DSS) for the emergency management of drinking water supply systems-infrastructuresexposed to extreme events. Specifically, $\mathrm{T}$ the DSS is based on the integration of: i) a probabilistic vulnerability assessment model, based on Bayesian Belief NetrorsBNs-(BBN), which is used-to identify the most critical elements of the eharacterize the infrastructural system-supporting in the identification of the critical elements; ii) an the associated uncertainty analysis-estimaterelated to the results of the vulnerability assessment model; iii) a BBN-based probabilistic-model for impact assessment; iv) the associated uncertainty estimate, useful to quantify the magnitude of impacts of an event. The most relevant innovation of the present work is twofold. Firstly, the definition of a methodology to perform $\underline{\text { a joint vulnerability and impact assessment of infrastructural failure, with an explicit uncertainty }}$ analysis. This is a crucial requisite in Athe definition of a joint analysis of set of decision-makers' preferences in emergency-to support defining-over the network attributes is proposed, in order to provide a ranking of the $\underline{\text { a priority of intervention-actions in emergency. Secondly, overcoming one }}$ of the main limits of BBNs, which are not inherently characterized by a spatial nature, $A \underline{a}$ GIS interface (G-Net) is-was also developedbuilt to support the management of input spatial information 
1 and results visualization. The DSS was developed and tested-with the cooperation of the Italian

2 Department of Civil Protection (DPC), tested and of with several Italian water utilities (Acquedotto

3 Pugliese S.p.A., Gran Sasso Acqua S.p.A. and AIMAG S.p.A.), and implemented - The DSS has been

4 then tested-in a relevant case study: L'Aquila (Italy) earthquake in 2009.

5 The paper is structured as follows. After the present introduction, Section 2 analyzes relevant 6 applicationsprovides an overview of $\mathrm{BBNs}$ features and applications in the field of emergency management for infrastructural systems, foeusing on the key potentialities and limits in decision making under uncertainty. Section 3 provides a description of describes the architecture of the

9 developed tool. Section 4 discusses the relevance of L'Aquila case study, while section 5 includes a discussion on the main results related to the implementation of $G$-Net, analyzing its potential and limitations.

\section{Methodological background: Bayesian Belief Networks}

$\mathrm{A} \mathrm{BBN}_{\underline{s}}$ combines graph theory and probability theory, consisting of a directed acyclic graph $\underline{s}$-and an-associated joint probability distribution (e.g. Pearl 1988-and Jensen 1996). The graph nodes represent variables, whereas the edges represent conditional dependencies. The strength of the dependency is represented by conditional probabilities: Eacheach node-variable $X_{i}$ is associated to a probability function $\underline{P\left(X_{i} \mid p_{a i}\right)}$ that takes as input $\underline{p}_{a i}$, i.e. a set of predecessors of $X_{i}$ which make $X_{i}$

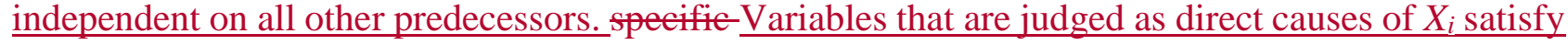
this property, and are the set of values for the node's parent variables of the node. and gives the probability of the variable represented by the node, thus defining the intensity of the dependency (Zhang et al. 2016).BBNs thus allow the probabilistic representation of interactions, which support to picture the relationships-between the-variables (Pearl 1988, Phan et al. 2016). The importance of BBNs is mainly related to the ability to coordinate bi-directional inferences, supporting the representation and analysis of uncertain knowledge as well as different modes of reasoning (Pearl 1988). 
1 BBNs have become an increasingly popular modelling technique to deal with complexity and 2 uncertainty and, particularly, several studies focused on the potentialities of BBNs to support 3 decision-making in different-several emergency conditions-Just to provide a few examples, BBNs 4 were used to describe the structure, uncertainty and losses of earthquake disaster chains ( (e.g. Wang 5 et al. 2013), to help volcano crisis management (Sobradelo et al. 2015, ) and to analyze natural gas 6 pipeline network accidents, supporting emergency operation (Wu et al. 2017). BBNs helped 7 overcoming the difficulties in decision making for water supply systems, particularly considering the 8 lack of information regarding their operation and failure conditions, supporting maintenance planning 9 (Mokhtar et al. 2016). A participatory BBN modelling approach was used to develop a risk assessment tool for estimating water quality-related health risks associated with extreme events (Bertone et al. 2016).

Within the field of emergency management $\underline{R}$, several successful applications of BBNs referring specifically to the analysis of-water supply infrastructures exposed to external stresses ${ }_{2}-$ BBNs were mainly used to build a-models for pipe breaks based onusing learning from past breaks-, integrating multiple kinds of data and modeling explicitly the dependencies, using probabilities updates and a representation of uncertainty (and covariate data, which proved insensitive to missing or incomplete data (Francis et al. 2014, ). A BBN based failure prediction models was proposed for water mains, integrating infrastructural features, soil information and pipe breakage data into a GIS (Kabir et al. 2015, f. Fuzzy Bayesian Belief Network were used by Kabir et al. (2016)for the safety assessment of oil and gas pipelines, due to their capability to model explicitly the dependencies of events, update probabilities and represent uncertain knowledge, thus strengthening decisions when empirical data are lacking.

$\underline{\text { A wide scientific literature underlined that BBNs are } \div \text { able to support: } ; \text { the integration of various }}$ types of information, (e.g. analytical models, expert knowledge, literature and historical data) $\underline{\text { (Gonzalez-Redin et al. 2016, Phan et al. 2016), } \div \text { the possibility of reasoning from uncertain evidence }}$ 
1 to uncertain conclusions (John et al. 2016), $\div$ the explicit treatment of uncertainties (Uusitalo 2007.

2 Uusitalo et al. 2015, Gonzalez-Redin et al. 2016). Furthermore, BBNs are also flexible enough to

3 support a revision of probabilities in the light of additional information or observations availability.

4 Shabarchin and Tesfamariam (2016) developed a BBN based model in GIS to assess internat

5 eorrosion for oil and gas pipelines, integrating also expert judgment. A decision support approach

6 based on Fuzzy Bayesian Networks was developed for assessing the conditions of existing pipelines

7 (Zhanget al. 2016). Bayesian Networks were used also to support water pipe leakage prediction (Let

8 and Bui 2016).

9 Bayesian approachesBBNs have also some limitations. Firstly, eөntinuøus variables are nøt easily

10

11 integrated within BBNs, leading often to-nodes that are often discretized with only a few states, $\underline{- \text { and }}$ in qualitative terms (e.g. 'high' or 'low'). These states might, provide providing enly a coarse representation of the node-(Uusitalo, 2007). Secondly, the BBNs structure of BBNs-is linear and static, and does not directly account for the analysis of feedback loops and dynamic issues (Uusitalo, 2007; Bertone et al. 2016). Furthermore, BBNs do not natively provide a spatial representation of variables.

Specifically referring to the last issue, Johnson et al. (2011) identified four main-ways to integrate

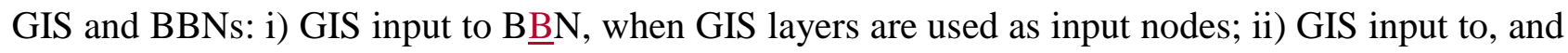
output from BBN, in case GIS is also used to visualize the output of a BBN; iii) BBN and GIS complex interactions, in case different layers of information from a GIS are combined; iv) BBN and GIS within a larger framework, where BBNs model one factor and GIS models other factors-in a larger system. Integrated methodologies based on on linking BBNs with-and GIS were recently proposed (e.g. Landuyt et al. 2015, Gonzalez-Redin et al. 2016, Molina et al. 2016, Liu et al. 2016), showing remarkable potentialities.

Referring to the most widely used BBNs software packages, none of them proposes meaningful ways to graphically represent the uncertainties associated to the output. Nevertheless, several $u$ Uncertainty 
1 maps can be developed as well-, as discussed by Landuyt et al. (2015)-compared standard deviation

2 maps, probability maps, sampled maps, ignorance maps, cumulative probability maps as techniques

3 to represent and analyze and represent uncertainty. Each one has its specific potentialities and

4 limitations, depending on type of output data, degree of uncertainty and objectives, final users.

\section{Model description}

The present work describes a DDS developed for decision-makers involved in the management of drinking water supply infrastructures under emergency conditions.

The DSS is based on the integration of:

- A probabilistic vulnerability assessment model, based on Bayesian Belief Networks (BBN), for the used to characterizinge the infrastructural system-performances in case of extreme events. The model is integrated in a GIS tool $(\mathrm{G}-\mathrm{Net})$ in order to facilitate data input and to provide a geographical visualization of results (Section 3.1).

- An uncertainty analysis related to the results of the vulnerability assessment model $\mathrm{L}_{-}$. It is based on the metries normally used with BBNs, and-used to analyze the impacts of the available knowledge (and existing gaps) on the results (Section 3.2).

__ A BBN-based probabilistic model for impact assessment, useful to quantify the magnitude of the impacts of an event (Section 3.3)..2.

- An uncertainty analysis related to the results of the impacts assessment model (Section 3.3).

In the end, decision-making is supported through the definition of a ranking order among the elements of the network, based on the integration of information on infrastructural vulnerability, related uncertainty and impacts and related uncertainties.

\subsection{Deseription of the tool ( G-Net) tool for the spatial vulnerability assessment}

The first element of the DSS is a vulnerability assessment tool for drinking water supply infrastructures based on BBN $\underline{s}$, whose conceptual structure is described in details-in Pagano et al. 
1 (2014aa). The tool is composed of a set of BBNs quantifying the vulnerability levels of drinking

2 water supply systems from source to tap, with respect to either physical (e.g.earthquakes, landslides)

3 or CBR hazards (water contamination). A couple of BBNs is thus associated to each subsystem of a

4 drinking water supply infrastructure.

5 The following Fig. 1 shows the BBN used to analyze the physical vulnerability of water mains-of

6 drinking water mains. It may be used either to assess the global vulnerability level, or the vulnerability

7 associated to specific mechanisms (i.e. breaking, corrosion, joint extraction and security level towards

8 human actions). The variables in grey represent the 'parent' variables (input), whereas those in yellow

9 are the 'child' variables (output).

The model is able to manage and integrate a wide range of data and information, belonging to $t$ Three main classes of data are included in the model: physical-infrastructural data- related to infrastructurat eharacteristics-(e.g. diameter, material, thickness, etc.); environmental data (e.g. seismicity, soil mechanical characteristics, etc.) and-; operative data (e.g. hydraulic variability, maintenance performed/scheduled, etc.). The outcome is, for each element of the network under investigation, a set of probability values associated to the states of ene or morespecific output variables (i.e. the globat physical vulnerability or the vulnerability associated to the specific mechanisms). Further details on $\underline{\text { model building are included in the Supplementary Material. }}$

\section{FIG 1}

Fig. 1 BBN used for the physical vulnerability assessment of water mains

One of the assumptions of the -It is worth mentioning that model (Pagano et al. 2014a) is that each element pipe of the whole infrastructural network is analyzed independently, thus neglecting the role of structural or functional interconnections, dependencies and cascading effects (e.g. the $\underline{a}$ vulnerability vulnerable element of an element-might have impacts on the whole infrastructure downstream that are neglected according to the present approach). This assumption is performed for the sake of simplicity, in order to easilyallows easily-identifying the most eriticat vulnerable elements of the whole network (further details in Pagano et al. 2014a). 
1 Several-Based on the feedbacks on model functioning were collected mainly interacting with obtained

2 by the potential end-users-of the tøel, i.e. Đept. of Civil Protection (DPC, the emergeney management

3 agency)_and water utilities, ${ }_{2}$. The main issues emerged are summarized in the following: i) a GIS

4 interface is neededwas built, in order to facilitate spatial data processing and the $\underline{\text { results spatial }}$

5 representation - of the results; ii) the a quantitative analysis of data and model uncertainty is crucial to

6 support decision-making in emergency; iii) the magnitude of impacts is a key driver for decision-

7 makers; iv) integrating and taking jointly into account all these aspect is not a straightforward process.

8 The model was thus developed following the above issues/suggestions, and a GIS based interface (G-

9 Net $)$ was built accordingly. Going further into details, $t$ The toolbox $(\underline{\underline{G}-\mathrm{Net}}) \underline{\underline{\text { consists of an expanded }}}$

10 development of a GIS application supporting the vulnerability assessment of drinking water supply

11 infrastructures, with data, models and user interfaces all integrated in GIS environmenttool. GNet It

12 is specifically designed to support the integration with Netica ${ }^{\mathrm{TM}}$ software by means of an automated

13 procedure-in which some typical GIS functions are organized in a specific workflow. The tool is

14 composed of customized interfaces working in ArcGIS® software (by Esri) environment with

15 wizards-specifically configured as interface between Netica ${ }^{\mathrm{TM}}$ and ArcGIS®.

16 The tool has been designed using open-source Python scripting language, fully supported by

17 ArcGIS $\AA$ and able to extend the basic functionality of GIS and to automate the workflow (Tateosian

18 2015). following aA loosely-coupled integration strategy between ArcGIS® and Netica ${ }^{\mathrm{TM}}$ was used.

19 This means that the latter is not completely encapsulated within a GIS environment-as in the tightly

20 eoupled appreach, but takes advantage of the database, the visualization and the analysis capabilities

21 of a GIS (Karimi and Houston 1996, Johnson et al. 2011)). From the technical point of view, the toot

22 has been developed in a GIS framework and customized using open-source Python scripting

23 language, fully supported by ArcGIS@ and able to extend the basic functionality of GIS and to

24 automate the workflow (Tateosian 2015). 
1 The global structure of the model is summarized in the following Fig. 2.

Figure 2. Conceptualization of the model and connection with spatial data for decision making

4 The toolbox for spatial analysis $(G$-Net $)$ was developed by IRSA CNR with a twofold objective.

5 Firstly, i) the toolbox should be usedboth for the collection, analysis and attribution of spatial input

6 data-with a spatial dimension to the variables of the model; ii). Secondly, and it is used tofor the

7 vistalize-visualization and mapping of the outcomes of the Bayesian-vulnerability assessment.

8 Referring to the different classes of BBN-GIS interactions introduced above (Johnson et al. 2011),

9 the developed toot $\underline{G-N e t}$ refers to the second category, which is 'GIS input to, and output from BBN'.

10

11

Going futher into detrils, the toolbox G- eonsists of an expded developent of a GIS applieation supporting the vulnerability assoment of drinking water supply infrastmetures, with data, models and en interfaces allinto sepport the integration with Netica ${ }^{\mathrm{TM}}$ software by means an alomat procedure in which some ypical GIS functions are organized in a specific workflow. The tol is mpor of sumized

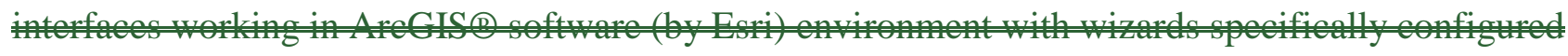
a inteen ${ }^{\mathrm{TM}}$ and AreGISO.

The tool has been designed following a loosely coupled integration strategy between AreGIS@ and Netica ${ }^{\mathrm{TM}}$ This means that the latter is nompletely encaptated within aGIS environment as in

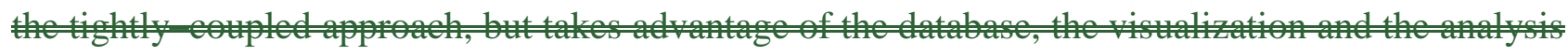
eapilitie of aIS (Karimind Hou 1996, John al. 2011). From an

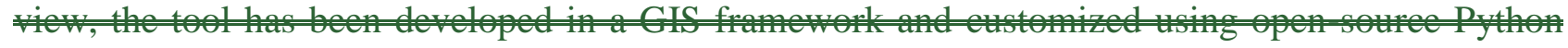
scripting languag, fully supported by AreGISQ and able to exend the basic functionality of GIS and toutemate the 2015 ). 
1 A schematic overview of the procedure carried out by the tool is shown in the followingthe Fig.ure 232.

\section{FIG $3 \underline{2}$}

Figure 32. G-Net procedure for vulnerability assessment and mapping: (a) selection of the analysis to perform; (b) data association to the input variables; (c) input variables export procedure; (d) output vulnerability map.

$G$-Net firstly requires the selection of the subsystem to analyze, among all the elements of a drinking water infrastructure, both linear (e.g. water mains) and punctual (e.g. tanks, pumping systems, etc.), available in vector data format (shapefile or features stored inside georeferenced database, both native data format for Esri software). Secondly, the user should select the kind of analysis to carry out (Figure $3 \mathrm{a} \underline{2 \mathrm{a}}$ ), i.e. physical or CBR vulnerability assessment. Additional data related to the input variables in the BBN can be manually or automatically associated to the file, either through an automatic overlay between the input vector and the available layers in the database, or through manuat

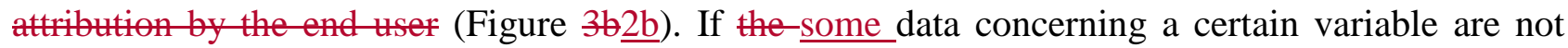
available, the user could attribute-a uniform probability distribution to the input data for this variableis considered and the - BBN propagates the information about the-related uncertainty up to the output variables. The tool allows end users also to define some variables using linguistic assessment, based en fuzzy sets (Pagano et al. 2014a).

Once the GIS pre-processing is complete, G-Net exports a table for the input variables in a format easily manageable by Netica ${ }^{\mathrm{TM}}$ (Figure $3 \mathrm{e} \underline{\mathrm{c}}$ ). Following the vulnerability assessment procedure in Netica $^{\mathrm{TM}}$, a table with modeling results can be imported again in GIS, and joined to the available file, through the same toolbox. Afterwards, the resulting BBN results can beis shown in the vulnerability map (Figure $3 \mathrm{~d} 2 \mathrm{~d}$ ). Additional functionalities are included in the toolbox, and an exhaustive help accompanies each step of the procedure. 


\subsection{Uncertainty analysis}

2 Estimating uncertainty is fundamental for effective decision-making. Such uncertainty may be either

3 related to the inherent structure of the model ('conceptual' uncertainty) or to information quality

4 ('data' uncertainty). Particularly the issue of 'data' uncertainty is crucial in emergency operations.

5 Understanding the quality and quantity of the available information, as well as how to improve it, is

6 erucial to improve decisions (Hsu et al. 2012).

7 The aim of the present section is toaims at defindefininge a waymethod to analyze and map the

8 uncertainty associated to the Bayesian vulnerability assessment modelBBNs, also-supporting the

9 identification of its root causes. Reference is made to the work by Marcot (2012), who suggested 10 metrics for estimating model performances and uncertainty. Referring to BBNs, uncertainty pertains 11 to the dispersion of Pposterior probability Probability 12 alternative predictions.

13 Firstly, the sensitivity analysis (SA) supports determining the degree to which a variation in PPD is explained by other variables, and-basically depicts the underlying probability structure of a model 15 (Marcot 2012, Pagano et al. 2014a). It was performed with respect to the variable 'breaking vulnerability', and the results are proposed in the following Table 1. The results of SA are also used (see section 5 for details), for scenario analysis (see section 5).

Table 1. Results of the sensitivity analysis performed with respect to the variable 'breaking vulnerability'

\begin{tabular}{lcccc}
\hline \multicolumn{1}{c}{ Node } & Mutual Info & Percent & Variance of Beliefs & Scenario \\
\hline Breaking Vulnerability & 1.3976 & 100 & 0.363296 \\
\hline External stress level & 0.19371 & 13.9 & 0.044494 \\
\hline Mechanical features & 0.09952 & 7.12 & 0.02237 \\
\hline Physical vulnerability & 0.04676 & 3.35 & 0.01062 & $(1),(3)$ \\
\hline Seismicity & 0.04403 & 3.15 & 0.010404 & $(1),(3)$ \\
\hline Existing instabilities & 0.02028 & 1.45 & 0.004848 & 0.004305 \\
\hline Actual conditions & 0.01908 & 1.37 &
\end{tabular}




\begin{tabular}{|c|c|c|c|c|}
\hline Soil mechanical characteristics & 0.01267 & 0.907 & 0.002837 & (3) \\
\hline Hydraulic efficiency & 0.01221 & 0.874 & 0.002945 & \\
\hline Safety level & 0.00808 & 0.578 & 0.001839 & \\
\hline Extra-maintenance & 0.0056 & 0.401 & 0.001275 & $(2),(3)$ \\
\hline $\mathrm{OP} / \mathrm{NP}$ & 0.00312 & 0.223 & 0.000758 & (2) \\
\hline Dynamic loads & 0.00269 & 0.193 & 0.000649 & (1) \\
\hline Flexibility & 0.00212 & 0.152 & 0.000485 & \\
\hline Hydraulic variability & 0.00138 & 0.0991 & 0.000338 & \\
\hline Age/Design life & 0.00111 & 0.0797 & 0.000256 & (2) \\
\hline Joint extraction vulnerability & 0.00084 & 0.0598 & 0.000204 & \\
\hline Maintenance: performed/scheduled & 0.00077 & 0.0548 & 0.000175 & (2) \\
\hline Joint type & 0.00063 & 0.0452 & 0.000145 & (2) \\
\hline Diameter & 0.00059 & 0.0422 & 0.000137 & (2) \\
\hline Depth & 0.0004 & 0.0283 & $9.49 \mathrm{E}-05$ & (2) \\
\hline Joint frequency & 0.00014 & 0.0102 & $3.25 \mathrm{E}-05$ & (2) \\
\hline Corrosion vulnerability & 0.00004 & 0.00251 & 0.000008 & \\
\hline Pipe coating & 0.00003 & 0.00235 & 7.9E-06 & \\
\hline Cathodic protection & 0.00001 & 0.000767 & $2.6 \mathrm{E}-06$ & \\
\hline Thrust restraint & 0 & 0 & 0 & (2) \\
\hline
\end{tabular}

2 Sensitivity is calculated with input variables set to uniform prior probability distributions (Marcot

3 2012) and supports in the identification of the most influential variables of the BBN. The more

4 sensitive to a variable the model is, the more important is to collect related information. Having

5 reliable data on key variables is a crucial requisite to reduce uncertainty.

6 Secondly, the uncertainty associated to BBNs is estimated using the Shannon entropy $H(X)$ referring

7 to the output variable ('breaking vulnerability' for the vulnerability assessment model). It is defined

8 as the average amount of information conveyed by a stochastic source of data. The concept of

9 Shannon Entropy is fundamental in information theory and, besides sharing some intuition with

10 Boltzmann's theory, some aspects are analogous to those used in statistical thermodynamics. The

11 Shannon entropy can be used as a synthetic measure of uncertainty, related to the number of

12 alternatives and characteristics of the probability distribution over the states of a random variable

13 (Das 1999). It is expressed as follows, using a logarithmic form:Secondly, the uncertainty associated 
1 to model predictions is estimated using the Shannon entropy $H(X)$. It can be used as a synthetic

2 measure of uncertainty, related to the number of alternatives and characteristics of the probability

3 distribution over the states of a variable (Das 1999). It is expressed as follows:

$$
H(X)=-\sum_{i=1}^{n} P\left(x_{i}\right) \log P\left(x_{i}\right)
$$

$H(X)$ measures the average information required in addition to the current knowledge to remove the

6 ignorance associated to the probability distribution of the variable $X$. Higher values of $H(X)$ are thus

7 associated to more uncertain decisions. If the current state of knowledge is complete, then $H(X)=0$.

8 If it is total ignorance (uniform probability distribution), the additional information required to pin down an alternative is maximum. A normalized value of entropy can be calculated as $\bar{H}(X)=$ $H(X) / H(X)_{\max }$. For the purposes of the present work, the Shannon entropy is used to estimate the

uncertainty related to the main output variables (i.e. 'breaking vulnerability' and 'impacts').The main advantages related to the use of the Shannon entropy instead of other metries, are the significance of information in case of skewed distributions and the absence of any influence of user defined thresholds.

\subsection{Impact assessment}

The levels and types of adverse impacts are the result of a physical event interacting with vulnerable elements. The aim of emergency managers is directly related to the reduction of impacts, both before and after a disaster occurs (McCormick 2016). Correctly assessing the impacts of an emergency is not a straightforward task, due to the complexity associated to a comprehensive analysis of costs and consequences (Sobradelo et al. 2015).

For the purpose of the present work, the impact assessment is performed through another $\mathrm{BBN}_{-}$,

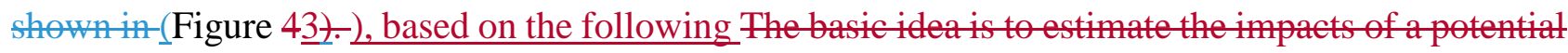
disfuption of the infrastructure identifying the -key driversvariables, namelydescribed in the

\section{following:}


- ${ }^{\text {a) }}$ 'Flow rate': measure of the service loss, depending on the number of users potentially affected. The values 'Hhigh', 'Mmedium' and 'Llow' are defined considering whether the $\underline{\text { ratio between the local flow rate and the maximum upstream value is higher than } 0.7 \text {, between }}$

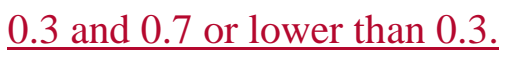

__ 'Diameter': measure of the cost for repair, proportional to pipe diameter. The values 'Hhigh', 'Mmedium' and 'Lolow' are defined for each element considering whether the ratio between the local diameter and the maximum value is higher than 0.7 , between 0.3 and 0.7 or lower than 0.3.

_ 'Relevance': defines the presence of critical users and services (e.g. hospitals). The values 'Hhigh', 'Mmedium' and 'Elow' are defined considering the importance of the services depending on the infrastructure.

_ 'Redundancy': defines the presence of additional paths for water supply. The values 'Yes' and 'No' are defined considering the presence of other paths that can be activated.

costs, both social (e.g. service loss) and economic (repair costs, proportional to diameter); b) relevance (i.e. potential critical users); c) redundancy (existence of alternative paths). $\Lambda$ more detailed description of the variables is in the following Table 2.

FIG $4 \underline{3}$

Figure $4 \underline{3}$. BBN for impact assessment

Table 2. Description of the variables used for impact assessment

\begin{tabular}{lll}
\hline Variable & \multicolumn{1}{c}{ Definition } & Description \\
\hline Flow rate & $\begin{array}{l}\text { Impact associated to the number of users } \\
\text { potentially affected. }\end{array}$ & $\begin{array}{l}\text { The values 'High', 'Medium' and 'Low' are defined } \\
\text { eonsidering whether the ratio between the local flow rate } \\
\text { and the maximum upstream value is higher than } 0.7, \\
\text { between } 0.3 \text { and } 0.7 \text { or lower than } 0.3 .\end{array}$ \\
\hline Diameter & $\begin{array}{l}\text { Defines the impacts of damages in terms } \\
\text { of costs for repair, proportional to the values 'High', 'Medium' and 'Low' are defined for } \\
\text { diameter. }\end{array}$
\end{tabular}


tocal diameter and the maximum value is higher than 0.7 ,

between 0.3 and 0.7 or lower than 0.3 .

\begin{tabular}{|c|c|c|}
\hline Relevance & $\begin{array}{l}\text { Defines the presence of critical users and } \\
\text { services (e.g. hospitals) }\end{array}$ & $\begin{array}{l}\text { The values 'High', 'Medium' and 'Low' are defined } \\
\text { considering the importance of the services depending on } \\
\text { the infrastructure. }\end{array}$ \\
\hline Redundaney & $\begin{array}{l}\text { Defines the presence of additional paths } \\
\text { for water supply. }\end{array}$ & $\begin{array}{l}\text { The values 'Yes' and 'No' are defined considering the } \\
\text { presence of other paths that can be activated (e.g. bypass). }\end{array}$ \\
\hline
\end{tabular}

\section{L'Aquila case study: relevance and main isstes}

3 L'Aquila province (central Italy) was struck by a severe earthquake on 6 April 2009. Apart from a

4 huge number of casualties, s $\underline{S}$ everal damages to structures and infrastructures were detected over a 5 broad area (Kongar et al. 2017). Referring specifically to the water supply system, the major damage 6 occurred on an important steel pipe (diameter $600 \mathrm{~mm}$; pressure $25-30 \mathrm{~atm}$ ), which failed because 7 crossing the surface trace of a fault activated during the earthquake (Dolce and Di Bucei 2017, Pagano 8 et al. 2017).

9 Emergency managers decided to stop $t$ The operation of the whole system was stopped; in order to 10 allow the restoration of infrastructural functionality and to limit the impacts of the multiplicity 11 efmultiple damages occurred in the urban distribution system. Nevertheless, this decision had a strong impact on the loeal community, whose aceess to-such acrucialservice was limited for some days.

According to the interviews held with technicians involved in emergency operations, the fragmented and uncertain knowledge related to infrastructural conditions, particularly in the urban area, was a key limit in during emergency operations in the aftermath of the disaster. Infrastructural data were not readily available, since most of information were unstructured and not accessible by operators. The available data were often not reliable and directly usable, since mainly deriving from personal experience, and thus difficult to share, visualize and integrate. Most of emergency operators acknowledged the lack of reliable infrastructural information as a main issue hampering the effectiveness of emergency management strategies. 
1 Based on the lessons learned in L'Aquila earthquake, the main potentialities of the proposed

2 integrated DSS to support decision-making on drinking water supply system in case of disasters are

3 investigated and described in the following.

5

7

\section{Results and discussion}

\subsection{Vulnerability assessment}

The main results of the vulnerability assessment procedure, performed through G-Net in L'Aquila case study, are represented in Figure 5(a) along with the results of the uncertainty assessment. These results are identified in the following as the following as 'BASE' scenario. The map plots the probability values associated to the state 'high' of the variable 'breaking vulnerability'.

The following Figure 5 (a) shows the presence of several elements having values of 'breaking vulnerability' from 'medium' to 'high'. Model predictions were tested comparing the results with the position of the main pipe breaks occurred during the earthquake. Particularly, tParticularly, $\underline{\mathrm{T}}$ the highest values of 'breaking vulnerability' were found for the pipe damaged in 2009. Then, other elements characterized by a significantly high 'breaking vulnerability' were identified as well, and the result discussed with GSA S.p.A., resulting in a with a positive outcome related to the identifieation ofcorrespondence with some well-known vulnerabilities of the infrastructure.

\section{FIG 5}

\section{Figure 5. Results of the vulnerability assessment model performed through $G$ Net}

Globally, the implementation of the model supports building a comprehensive knowledge framework on the conditions of the infrastructure, thus identifying its main criticalities. Although the model is primarily meant to support emergency management activities, it can be used for ordinary operation as well (e.g. to prioritize and schedule maintenance). 


\subsection{Uncertainty analysis and mapping}

2 Starting from the results of the sensitivity analysis $\underline{S A}$ proposed in the $($ Section 3.2), an influence

3 analysis was performed. It allows evaluating (and comparing) the effects on PPD from selected input

$4 \quad$ variables set to specific scenario values-(generally best or worst cases). Conducting influence runs

5 can help reveal the degree to which individual or sets of input variables could affect output

6 probabilities. This is helpful in a decision--setting, where management might prioritize activities to

7 best effect desirable, or to avoid undesirable outcomes (Marcot 2012).

8 The following scenarios were analyzed and are discussed in the following:

- BEST Scenario: the scenario is built setting all the variables to their optimal state - i.e. minimizing the vulnerability of the system.

- WORST Scenario: the scenario is built setting all the variables to their worst state - i.e. maximizing the vulnerability of the system.

- UNCERTAIN Scenario: the scenario is built setting all the variables to an 'unknown' state i.e. the input variables have all an-uniform probability distribution, in case no information is available.

Three additional scenarios were built as well, changing the state of some variables according to the results of the SA. The variables modified in each scenario are identified in the Table 1.

- SENSIT (1). The scenario is built setting three key environmental variables to the worst state: 'seismicity', 'existing instabilities' and 'dynamic loads', which are among the most influential variables on 'breaking vulnerability'. All the variables considered in this scenario represent external conditions, and thus their state cannot be improved.

- SENSIT (2). The scenario is built considering the positive impact of actions performed on variables that can be modified through specific strategies. These variables may be 
representative of both structural and operational aspects. In this scenario, a subset of variables is set to the best state.

- SENSIT (3). The scenario is built considering the four most influential variables, according to the sensitivity analysis, all eontextually-set to the worst state.

The results are summarized (according to Marcotet al. 2012), in terms of PPD of the output variable 'breaking vulnerability' (Figure 64). The 'BEST', 'WORST' and 'UNCERTAIN' scenarios show an intuitive PPD for the output variable. The comparison between the scenarios 'SENSIT (3)' and 'SENSIT (1)' suggest that few variables, mainly related to environmental conditions, are highly influential on the result. From a practical point of view, this means that a deep knowledge of the environment in which a system is located (e.g. seismicity of the area, existing instabilities) is crucial for providing athe reliable estimate of 'breaking vulnerability'. Nevertheless, these variables cannøt be modified or significantly conditioned. The Scenario 'SENSIT (2)' is indeed relevant in order to assess the impact of potential improvements on infrastructural and operational features, which can be modified. Although the effect on the output PPD is lower, acting on the infrastructure (both through design and maintenance) and changing operative conditions may contribute to reduce significantly the vulnerability level of the system.

\section{FIG $6 \underline{4}$}

Figure $6 \underline{4}$. Results of the influence analysis in the modeled-scenarios

The Shannon entropy was then used to produce uncertainty maps, as shown in Fig. 5. It was firstly used in-Referring to the 'BASE' scenario, focusing on the main output variable, i.e. the 'breaking vulnerability', as a simple measure of the uncertainty related to model results. Tthe values of $H(X)$ were computed for the whole network and spatially plotted along with the results of the vulnerability assessment (Fig. 5a), in order to describe the spatial variation of uncertainty. The same procedure was This coupling (Figure 7) supports the identification of the most critical elements of the system (e.g. 
1 high vulnerability associated with low uncertainty) and the areas where additional information would

2 be primarily beneficialused to map the impacts magnitude and the related uncertainty (Fig. 5b).

3 Decision-makers can be thus supported to schedule (and prioritize) actions and to identify locations

4 where additional data and investigation would be worth.

Figure 7. Coupled spatial representation of model results and related uncertainty

7 The relevance of the Shannon entropy $H(X)$ for uncertainty assessment was further tested through

8 specific simulations, analyzing the impacts of the lack of important input information on the reliability

9 of model results. The selection of the input variables to be considered in such analysis, was performed according to the sensitivity analysis.

11 The 'BASE' Scenario was built considering a full knowledge of the input variables required by the model. Referring also to Table 1, the following scenarios were created:

- U-(1)_Scenario $\div$ this scenario was built considerings complete uncertainty for the input variables identified with (1) in Table 1. Particularly, $\underline{T}$ three highly influential (according to the sensitivity analysis) environmental variables (according to the SA):-i.e. 'seismicity', 'existing instabilities' and 'dynamic loads', are set to a uniform probability distribution, that is they are treated as unknown.

- U-(2):- Scenario this scenario was built consideringconsiders complete uncertainty for the input variables identified with (2) in Table 1. Both structural and operative features are set to a uniform probability distribution.

- U-(3)_Scenario: this scenario was built_eonsidering considers uncertainty for the input variables identified with (3) in Table 1. In this case, and the four most relevant variables according to the SA are set as unknown. 
1 The Shannen entropy $H(X)$ was used - in the cited scenarios, to quantify the cumulative uncertainty

2 related to unknown inputs. Following the 'chain rule' for entropy, the global entropy of a group of

3 random variables was computed as the sum of conditional entropies. The values of $H(X) \underline{\text { Shannon }}$

4 entropy are $0,0.067,0.012$ and 0.083 respectively for BASE, U(1), U(2) and U(3) scenarios. This $A$

5 summary of the results is proposed in the following Table 3:

Table 3. Results of the Shannon entropy for the cited scenarios

\begin{tabular}{cc}
\hline Scenario & Shannonentropy (input variables) \\
\hline BASE & $\theta$ \\
$U(1)$ & 0.067 \\
$U(2)$ & 0.012 \\
$U(3)$ & 0.083 \\
\hline
\end{tabular}

7 The outcomes of this uncertainty analysfirstlyThis -suggests that although the scenario U-(2) is

8 characterized by a higher number of unknown variables, their impact on modeling results is lower if

9 compared to the key variables neglected in both U-(1) and U-(3) scenarios. Both U-(1) and U-(3) scenarios suggest that the knowledge related to environmental conditions is a key requirement to

11 perform a reliable vulnerability assessment. Furthermore, referring particularly to the scenario U-(3), 12 the highest value of the Shannen entropy $\underline{H(X)}$ is representative of a more critical condition, due to 13 the highly uncertain set of available input data.

\subsection{Impact assessment}

15 The results of the impact assessment can be geographically-represented, as in the following Figure

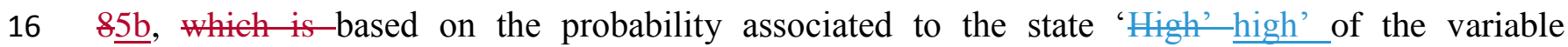
'Impacts'impacts'. Both a numerical and a chromatic scale are used. It is worth to remind that the impacts associated to the pipes actually occur downstream, in the urban areaAs already discussed, the map represents also the associated uncertainty. 


\section{$5 \quad 5.4$ Recommendations for decision-makingmakers} modelling results are jointly taken into account. uncertainty is a key parameter to be taken into account. of attributes $\mathcal{A}=\left\{\alpha_{1}, \alpha_{2}, \alpha_{1 u}, \alpha_{2 u}\right\}, \quad$ such $\quad$ that $\mathcal{A}_{\mathcal{L}}=$ of attributes $\mathcal{A}=\left\{\alpha_{1}, \alpha_{2}, \alpha_{1 u}, \alpha_{2 u}\right\}, \quad$ such that $\mathcal{A}_{\mathcal{L}}=$ b) Results of impacts assessment and related uncertainty.

Integrating the results already described, the aim of the The present section is toaims at supporting the decision-makers in prioritizing the interventions on a drinking water supply infrastructure, aiding in the definition of strategies in emergency management operations and to reduce the main eriticalities. The-specific values of infrastructural vulnerability, the magnitude of the expected impacts-associated to a potential failure, and the role of data and information-uncertainty related to

In order to address the problem of ranking among the network elementsMore specifically, The network elements alternatives to beare compared represent conditions where-consideringa different combinations of 'vulnerability under uncertainty' and 'potentialimpacts under uncertainty' are found, e.g. highly vulnerable elements of the network, having potentially high associated impacts are by far more relevant for a decision-maker than elements with low vulnerability and low impacts. Nevertheless, intermediate situations need a more careful assessment, also considering that results

Considering the drinking water supply infrastructure under analysis, we denote $X=\left\{x_{ \pm}, \ldots, x_{n}\right\}$ the set network elements $(n=254)$. Each-, each network element_ $(n=254)$ is characterized by the set

$22\left\{v_{h}, v_{m}, v_{l}, e_{h}, e_{m}, e_{l}, u_{1 h}, u_{1 m}, u_{1 l}, u_{2 h}, u_{2 m}, u_{2 l},\right\}$ represents the set of all possible values that the 23 elements of $\mathcal{A}$ can take, over which a decision-maker has preferences. Specifically;-the attributes are: 
$1-\alpha_{1}$, vulnerability based on the state 'high' of the variable 'breaking vulnerability'. The possible

$2 \quad$ values of the attribute are $\alpha_{1}=\left\{\operatorname{high}\left(v_{h}\right)\right.$, medium $\left(v_{m}\right)$, low $\left.\left(v_{l}\right)\right\}$;

$3-\alpha_{2}$, impact assessment through the analysis of the exposure to the potential effects of failures

$4 \quad$ represented by the values $\alpha_{2}=\left\{\operatorname{high}\left(e_{h}\right)\right.$, medium $\left(e_{m}\right)$, low $\left.\left(e_{l}\right)\right\}$;

$5-\alpha_{1 u^{-}}$and $\alpha_{2 u}$ respectively uncertainty associated respectively to vulnerability and to the impact 6 assessment, according to the values of the normalized Shannon entropy $\bar{H}(X), \alpha_{1 u}=$ $7 \quad\left\{\operatorname{high}\left(u_{1 h}\right)\right.$, medium $\left(u_{1 m}\right)$, low $\left.\left(u_{1 l}\right)\right\}$

$8 \quad$ and $\alpha_{2 u}=\left\{\operatorname{high}\left(u_{2 h}\right) \text {, medium }\left(u_{2 m}\right) \text {, low }\left(u_{2 l}\right)\right\}_{\text {. }}$

9 Throughout this section, the symbol $>$ denotes a decision maker's preference relation, $x>y$ means that $x$ is preferred to $y$ for one or more criteria considered all together. . The decision-makers have the following order of preferences: -a higher value of vulnerability-lexposure has priority compared to a lower one: $\left.t_{-} v_{h}>v_{m}>v_{l}\right)$ and a higher value of exposure has priority compared to at lower value $\left(e_{h}>e_{m}>e_{l}\right)$. . TThe rankingpreferences elicitation was performed through S $\underline{\text { semi- }}$ structured interviews were-held with Civil Protection operators and with-engineers working for the local water utility. They were asked, according to their experience in emergency management operations, to support in the ranking among the attributes. CConsidering the combination between the two attributes, $\alpha_{ \pm}$and $\alpha_{z}$, the decision-makers should prioritize the highest possible value of $\alpha_{1}$ combined with the highest possible value of $\alpha_{2}: v_{h} e_{h}>v_{h} e_{m}>v_{m} e_{h}>v_{h} e_{l}>v_{m} e_{m}>v_{l} e_{h}>$ $v_{m} e_{l}>v_{l} e_{m}>v_{l} e_{l}$. However, as discussed in section 5.2, the 'uncertainty' $\alpha_{1 u}$ is a key attribute that decision-makers take into account. No matter theConsidering the preferences on the other eonditionsattributes, a lower value of the 'uncertainty' associated respectively to vulnerability and

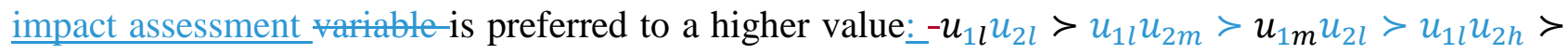
$u_{1 m} u_{2 m}>u_{1 h} u_{2 l}>u_{1 m} u_{2 h}>u_{1 h} u_{2 m}>u_{1 h} u_{2 h}$ and $u_{t}>u_{m}>u_{h}$. 


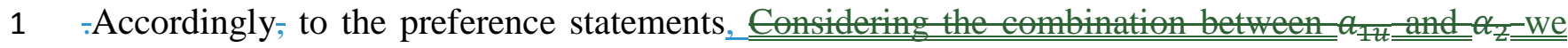

2 obtain the following compact preferences-representation- supporting the definition of a ranking order

3 among the different potential 81 conditions, we get:

4

5

6

7

8

9

10

11

12

13
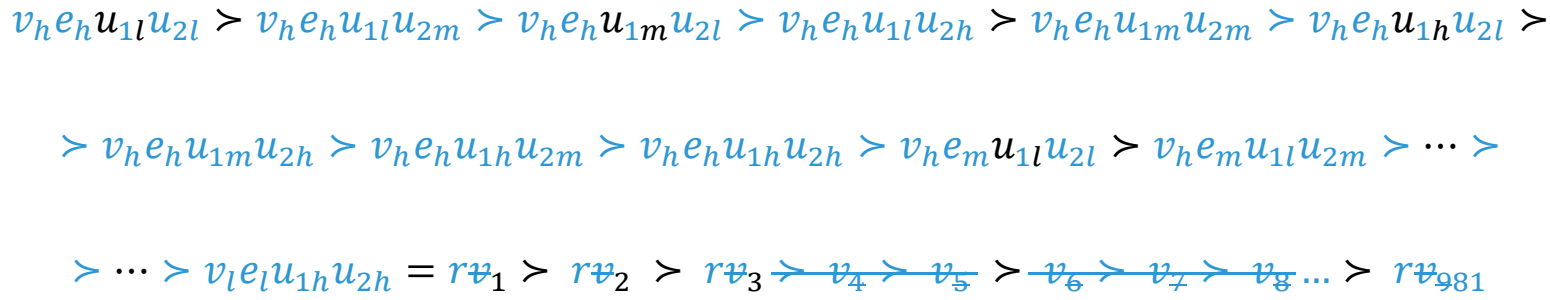

$v_{n} u_{t}>v_{n} u_{m}>v_{m} u_{t}>v_{n} u_{n}>v_{m} u_{m}>v_{t} u_{t}>v_{m} u_{n}>v_{t} u_{m}>v_{t} u_{n}$ C Considering the

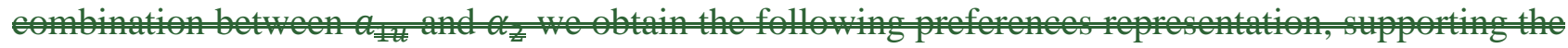
definition of a ranking order among the different pontial onditions-

Consequentially, eonsidering thein relation to the water supply network under analysis, we obtain the spatial representation of ranking as in the following Figure. $9 \underline{6}$. The mapping of results allows decision-makers to identify the elements of a complexthe network where interventions should be primarily oriented either in emergency conditions or in ordinary management, to reduce the risk levels for the whole system. With respect to the results of the vulnerability assessment, proposed in Figure 5 according to the methodology by Pagano et al. (2014a, 2014b), the present approach provides an added value for decision making processes, since the final ranking takes into account the uncertainty of modeling results, and the magnitude of impacts.

\section{FIG $9 \underline{6}$}

Figure $9 \underline{6}$. Ranking of the network elements-of the network. Priority decreases from elements belonging to $r_{3}$ to those belonging to $r_{z 0^{\circ}}$ 
1 This work describes the development of a Decision Support ToolDSS for decision-makers-making

2 involved in the emergency management of drinking water supply systems, in case of extreme events.

3 The Modelling methodology activities were carried out in tight cooperation with both the Italian

4 Bepartment of Civil Protection and the tool-was implemented in L'Aquila earthquake case study. The

5 model is composed of: i) a BBN-based vulnerability assessment tool for drinking water supply

6 infrastructures, with the related; ii) an-uncertainty analysis tool; iii) and a BBN-based model to

7 estimate impacts magnitude, in terms of both economic consequences and service limitationwith the

8 related uncertainty analysis. The tools are integrated in a comprehensive methodology, based on

9 preferences orders, capable to jointly take into account all the previous information, and to define a

10 ranking order among the elements of the infrastructural system. This ranking simply suggests a

11 priority of action for decision-makers. Overcoming one of the main limitations of BBNs -i.e. the

12 difficulties in performing spatial analyses- the development of a GIS interface ( $G$-Net), usedfor data

13 structuring and results analysis, revealed highly useful to improve the effectiveness of the tool,

14 helping in visualizing the outcomes, understanding the related-quantifying uncertainty, and

15 identifying the final ranking. Future activities will be oriented mainly to the analysis of temporal

16 aspects related to the dynamic evolution of system behavior (see e.g. Pagano et al. 2017) and to the

17 implementation of models based on complexity theory to support the analysis of interconnected

18 systems.

\section{Acknowledgments}

21 The present research activity was developed within a research project funded by the Italian

\section{References}


1 Bagheri A, Darijani M, Asgary A., Morid S. (2010) Crisis in Urban Water Systems during the

2 Reconstruction Period: A System Dynamies Analysis of Alternative Policies after the 2003

3 Earthquake in Bam-Iran. Water Resour Manage 24:2567-2596. doi:10.1007/s11269-009-9568-1

4 Beh EHY, Zheng F, Dandy GC, Maier HR, Kapelan Z (2017) Robust optimization of water

5 infrastructure planning under deep uncertainty using metamodels, Environ Model Softw 93:92 105.

6 doi:10.1016/j.envsoft.2017.03.013.

7 Bertone E, Sahin O, Richards R, Roiko A (2016) Extreme events, water quality and health: A

8 participatory Bayesian risk assessment tool for managers of reservoirs. J Clean Prod 135:657-667.

$9 \quad$ doi:10.1016/j.jelepro.2016.06.158

Das B (1999) Representing Uncertainties Using Bayesian Networks. DSTO-TR-0918, DSTO Electronics and Surveillance Research Laboratory, Australia

Diao K, Sweetapple C, Farmani R, Fu G, Ward S, Butler D (2016) Global resilience analysis of water distribution systems. Water Res 106:383-393. doi.org/10.1016/j.watres.2016.10.011

Bolce M, Di Bucci D (2017) Comparing recent Italian earthquakes. Bull Earthq Eng 15(2): 497-533. doi:10.1007/s10518-015-9773-7

Eidsvig UMK, Kristensen K, Vangelsten BV (2017) Assessing the risk posed by natural hazards to infrastructures. Nat Hazards Earth Syst Sci 17:481-504. doi:10.5194/nhess-17-481-2017. EPA (2015) Systems Measures of Water Distribution System Resilience. EPA 600/R-14/383.

Fragiadakis M, Christodoulou SE, Vamvatsikos D (2013) Reliability Assessment of Urban Water

Distribution Networks Under Seismic Loads. Water Resour Manage 27: 3739-3764. doi:10.1007/s11269-013-0378-0 
1 Francis RA, Guikema SD, Henneman L. (2014) Bayesian Belief Networks for predicting drinking

2 water distribution system pipe breaks. Reliab Eng Syst Saf 130:1-11. doi:

$3 \quad$ 10.1016/j.ress.2014.04.024.

4 Gaudard L, Romerio F (2015) Natural hazard risk in the case of an emergency: the real options' 5 approach. Nat Hazards 75(1): 473-488. doi:10.1007/s11069-014-1330-1

6 Giordano R, D’Agestino D, Apollonio C, Scardigno A, Pagano A, Portoghese I, Lamaddalena N,

7 Piccinni AF, Vurro M (2015) Evaluating acceptability of groundwater protection measures under

8 different agricultural policies. Agr Water Manag 147:54-66. doi: 10.1016/j.agwat.2014.07.023.

9 Gonzalez-Redin J, Luque S, Poggio L, Smith R, Gimona A (2016) Spatial Bayesian belief networks as a planning decision tool for mapping ecosystem services trade-offs on forested landscapes. Environ Res 144:15-26. doi: 10.1016/j.envres.2015.11.009.

Hsw WK, Tseng CP, Chiang WL, Chen CW (2012) Risk and uncertainty analysis in the planning stages of a risk decision-making process. Nat Hazards 61(3):1355-1365. doi:10.1007/s11069-01100321 Jessen F.(1996) An Introduction of Bayesian Network, Springer Verlag.

John A, Yang Z, Riahi R, Wang J (2016) A risk assessment approach to improve the resilience of a seaport system using Bayesian networks. Ocean Eng 111:136-147. doi: 10.1016/j.oceaneng.2015.10.048. Information Systems: Good Practice Examples. Integr Environ Assess Manag 8(3): 473-479. doi: 
1 Kabir G, Demissie G, Sadiq R, Tesfamariam S (2015) Integrating failure prediction models for water

2 mains: Bayesian belief network based data fusion. Knowl Based Syst 85:159-169. doi.

$3 \quad 10.1016 /$ j.knosys.2015.05.002

4 Kabir G, Sadiq R, Tesfamariam S (2016) A fuzzy Bayesian belief network for safety assessment of $5 \quad$ oil and gas pipelines. Struct Infrastruct Eng 12(8):874-889. doi:10.1080/15732479.2015.1053093

6 Karimi HA, Houston BH (1996) Evaluating strategies for integrating environmental models with 7 GIS: current trends and future needs. Comput Environ Urban Syst 20(6):413-425. doi: $8 \quad 10.1016 / \mathrm{S} 0198-9715(97) 00006-9$

9 Kongar I, Esposito S, Giovinazzi S (2017) Post-earthquake assessment and management for infrastructure systems: learning from the Canterbury (New Zealand) and L'Aquila (Italy) earthquakes. Bull Earthq Eng 15(2): 589-620. doi: 10.1007/s10518-015-9761-y

Landuyt D, Broekx S, D'hondt R, Engelen G, Aertsens J, Goethals P (2013) A review of Bayesian belief networks in ecosystem service modelling. Environ Model Softw 46:1-11. doi: 10.1016/j.envsoft.2013.03.011

Landuyt D, Van der Biest K Broekx S, Staes J Meire P, Goethals PLM (2015) A GIS plug-in for uncertainties in ecosystem service mapping. Environ Model Softw 71:30-38. doi: 10.1016/j.envsoft.2015.05.002.

Leu SS, Bui QN (2016). Leak Prediction Model for Water Distribution Networks Created Using a

Bayesian Network Learning Approach. Water Resour Manage 30:2719-2733. doi:10.1007/s11269-

\section{6-1316-8}

Liu R, Chen Y, Wu J, Gao L, Barrett D, Xu T, Li L, Huang C, Yu J (2016) Assessing spatial likelihood of flooding hazard using naive Bayes and GIS: a case study in Bowen Basin, Australia. Stoch Environ 
1 Mala-Jetmarova H, Sultanova N, Savic D (2017) Lost in optimisation of water distribution systems?

2 A literature review of system operation, Environ Model Softw 93:209-254. 3 doi:10.1016/j.envsoft.2017.02.009.

4 Malm A, Moberg F, Rosén L., Petterson TJR (2015) Cost Benefit Analysis and Uncertainty Analysis 5 of Water Loss Reduction Measures: Case Study of the Gothenburg Drinking Water Distribution 6 System. Water Resour Manage 29(15): 5451-5468. doi:10.1007/s11269-015-11282

7 Marcot BG (2012) Metrics for evaluating performance and uncertainty of Bayesian network models.

$8 \quad$ Ecol Model 230:50-62. doi: 10.1016/j.ecolmodel.2012.01.013

9 McCormick S (2016) New tools for emergency managers: an assessment of obstacles to use and implementation. Disasters 40(2): 207-225. doi: 10.1111/disa.12141.

Møhajerani H, Khølghi M, Møsaedi A, Farmani R, Sadoddin A, Casper M (2017)-Application of Bayesian Decision Networks for Groundwater Resources Management Under the Conditions of High Uncertainty and Data Searcity. Water Resour Manage 31(6):1859-1879. doi:10.1007/s11269-017 $1616-7$

Mokhtar EHA, Laggoune R, Chateamneuf A. (2016) Utility Based Maintenance Optimization for Complex Water Distribution Systems Using Bayesian Networks. Water Resour Manage 30:41534170. doi:10.1007/s11269-016-14129

Molina JL, Farmani R, Bromley J (2011) Aquifers management through evolutionary bayesian networks:the Altiplano case study (SE Spain). Water Resour Manag 25(14):3883-3909. doi:10.1007/s11269-011-9893-Z

1 Molina JL, Zazo S, Rodríguez-Gonzálvez P, González-Aguilera D (2016) Innovative Analysis of Runoff Temporal Behavior through Bayesian Networks. Water 8(11), 484. doi:10.3390/w8110484 
1 Pagano A, Giordano R, Portoghese I, Fratino U, Vurro M (2014a) A Bayesian vulnerability assessment tool for drinking water mains under extreme events. Nat Hazards 74(3):2193-2227. doi: 10.1007/s11069-014-1302-5

Pagano A, Giordano R, Portoghese I, Vurro M, Fratino U (2014b) Emergency Management of Drinking Water Infrastructures Based on a Bayesian Decision Support System. Vulnerability, Uncertainty, and Risk: Quantification, Mitigation, and Management - Proceedings of the 2nd International Conference on Vulnerability and Risk Analysis and Management, ICVRAM 2014 and the 6th International Symposium on Uncertainty Modeling and Analysis, ISUMA 2014, pp. 20122021.

Pagano A, Pluchinotta I, Giordano R, Vurro M (2017) Drinking water supply in resilient cities: notes from L'Aquila earthquake case study. Sustain Cities Soc 28:435-449. doi: 10.1016/j.scs.2016.09.005.

Pearl J (1988) Probabilistic Reasoning in Intelligent Systems, Morgan Kaufmann, San Francisco

Perng SY, Buscher M (2015) Uncertainty and Transparency: Augmenting Modelling and Prediction for Crisis Response, Proceedings of the ISCRAM 2015 Conference, Kristiansand, May 24-27, Palen, Büscher, Comes \& Hughes eds.

Phan TD, Smart JCR, Capon SJ, Hadwen WL, Sahin O (2016) Applications of Bayesian belief networks in water resource management: A systematic review. Environ Model Softw 85:98-111. doi: 10.1016/j.envsoft.2016.08.006

Refsgaard JC, van der Sluijs JP, Højberg AL, Vanrolleghem PA (2007) Uncertainty in the environmental modelling processe. A framework and guidance. Environ Model Softw 22:1543-1556. Shabarchin O, Tesfamariam S (2016) Internal corrosion hazard assessment of oil \&gas pipelines using Bayesian belief network model. J Loss Prev Process Ind 40:479-495. doi: 10.1016/j.jlp.2016.02.001 
1 Sobradelo R, Martı J, Kilburn C, Lopez C (2015) Probabilistic approach to decision-making under uncertainty during volcanic crises: retrospective application to the El Hierro (Spain) 2011 volcanic crisis. Nat Hazards 76:979-998. doi: 10.1007/s11069-014-1530-8

4 Sword Daniels V, Eriksen C, Hudson Doyle EE, Alaniz R, Adler C, Schenk T, Vallance S (2016)

5 Embodied uncertainty: living with complexity and natural hazards. J Risk Res. doi:

$6 \quad 10.1080 / 13669877.2016 .1200659$

7 Tanyimboh TT (2017) Informational Entropy: a Failure Tolerance and Reliability Surrogate for

8 Water Distribution Networks. Water Resour Manage 31:3189-3204. doi:10.1007/s11269-017-168498

10

Tateosian L (2015) Python For ArcGIS. Springer. doi: 10.1007/978-3-319-18398-5

Uusitalo L (2007) Advantages and challenges of Bayesian networks in environmental modeling. Ecol Model 203(3-4):312-318. doi:10.1016/j.ecolmodel.2006.11.033

Uusitalo L, Lehikoinen A, Helle I, Myrberg K (2015) An overview of methods to evaluate uncertainty of deterministic models in decision support. Environ Model Softw 63:24-31. doi: 10.1016/j.envsoft.2014.09.017

van der Keur P, van Bers C, Henriksen HJ, Nibanupudi HK, Yadav S, Wijaya R, Subiyono A, Mukerjee N, Hausmann HJ, Hare M, van Scheltinga CT, Pearn G, Jaspers F (2016) Identification and analysis of uncertainty in disaster risk reduction and climate change adaptation in South and Southeast Asia, Int J Disaster Risk Reduct 16: 208-214. doi:10.1016/j.ijdrr.2016.03.002

Wang J, Gu X, Huang T (2013) Using Bayesian networks in analyzing powerful earthquake disaster ehains. Nat Hazards, 68(2):509-527. doi:10.1007/s11069-013-0631-0 
1 Wu J, Zhou R, Xu S, Wu Z (2017) Probabilistic analysis of natural gas pipeline network accident

2 based on Bayesian network, Journal of Loss Prevention in the Process Industries, 46:126-136.

3 doi:10.1016/j.jlp.2017.01.025.

4 Zhang L, Wu X, Qin Y, Skibniewski MJ, Liu W (2016). Risk Anal 36(2):278 301, doi: $5 \quad 10.1111 /$ risa. 12448

6 Zhao X, Cai H, Chen Z, Gong H, Feng Q (2016) Assessing urban lifeline systems immediately after 7 seismic disaster based on emergency resilience. Struct Infrastruct Eng 12(12):1634-1649. doi: $8 \quad 10.1080 / 15732479.2016 .1157609$ 